BUTP-96/18

\title{
Lagrangian and Hamiltonian Formalism for Discontinuous Fluid and Gravitational Field
}

\author{
P. Hájíček \\ Institute for Theoretical Physics \\ University of Bern \\ Sidlerstrasse 5, CH-3012 Bern, Switzerland \\ J. Kijowski \\ Center for Theoretical Physics \\ Polish Academy of Sciences \\ Aleja Lotników 32/46, 02-668 Warsaw, Poland \\ and \\ Department of Mathematical Methods in Physics \\ University of Warsaw \\ Ul. Hoża 74, 00-682 Warsaw, Poland
}

July 1997

\begin{abstract}
The barotropic ideal fluid with step and $\delta$-function discontinuities coupled to Einstein's gravity is studied. The discontinuities represent star surfaces and thin shells; only non-intersecting discontinuity hypersurfaces are considered. No symmetry (like eg. the spherical symmetry) is assumed. The symplectic structure as well as the Lagrangian and the Hamiltonian variational principles for the system are written down. The dynamics is described completely
\end{abstract}


by the fluid variables and the metric on the fixed background manifold. The Lagrangian and the Hamiltonian are given in two forms: the volume form, which is identical to that corresponding to the smooth system, but employs distributions, and the surface form, which is a sum of volume and surface integrals and employs only smooth variables. The surface form is completely four- or three-covariant (unlike the volume form). The spacelike surfaces of time foliations can have a cusp at the surface of discontinuity. Geometrical meaning of the surface terms in the Hamiltonian is given. Some of the constraint functions that result from the shell Hamiltonian cannot be smeared so as to become differentiable functions on the (unconstrained) phase space. Generalization of the formulas to more general fluid is straifgtforward. 


\section{Introduction}

Spherically symmetric thin shells or dust stars (like the Oppenheimer-Snyder one) are popular models used extensively in the study of a number of phenomena: properties of classical gravitational collapse [1], properties of classical black holes [2], quantum gravitational collapse [3], the dynamics of domain walls in early Universe [4], the back reaction in Hawking effect [5], entropy on black holes [6] or quantum theory of black holes [7], [8], to mention just few examples.

The classical dynamics of objects with discontinuities in matter density is wellunderstood; it is determined by Einstein's equations, the matter dynamical equations and some jump conditions at the discontinuity. The jump conditions for the step-like discontinuity require that there are coordinates in which the metric is $C^{1}$ at the discontinuity surface [9]; for the thin shells, they have been first formulated by Dautcourt [10]; Dautcourt's equations have been rewritten in a covariant form by Israel [11.

In many investigations, however, a variation principle, or a Hamiltonian is needed from which this classical dynamics follows. Often, such principles (suitable eg. for spherically symmetric models) are just guessed from the dynamical equations; some attempts to obtain them from more general variational principles are [12] and [5]. Indeed, this is an interesting problem by itself: how the large number of different one-degree-of-freedom Hamiltonians scattered in literature is related to the EinsteinHilbert action? For our overal picture of the world has to be self-consistent, even if we indulge in using a number of different models, each just applicable for a situation under study.

In the present paper, we reformulate the dynamics of gravitation and ideal discontinuous fluid in the Hamiltonian form. That is, we identify the canonical variables ( $p$ 's and $q$ 's) and Lagrange multipliers and write down a Hamiltonian functional of these variables; we show that the constraints and the canonical equations resulting from this Hamiltonian are equivalent to the system of Einstein equations and the ideal fluid dynamical equations (plus the Israel equations in the case of a thin shell).

To identify the suitable symplectic structure and find the variational formulas, we employ the methods described in detail in 13 and their application to general relativity as given in [14. We will, however, keep the paper self-contained by motivating and explicitly performing all relevant derivations.

The model of matter used extensively in this paper is that of the simplest kind: the barotropic ideal fluid. This can be formulated as a Lagrangian field theory

without any constraints [15]. Generalization to ideal fluid with internal degrees of freedom (such as [16]) or to any conservative continuum should be straightforward; in any case, the gravitational parts of our Lagrangians and Hamiltonians (which represent the solution to the main problem) have general validity. 
In each particular case, the classical dynamics can be obtained from a variational principle that has the same form as the corresponding variational principle for a smooth system, if some particular generalized functions are allowed to describe the matter distribution: the step function for star boundaries and the $\delta$-function for thin shells (cf. [12]). This simplicity is, however, traded for the freedom in the choice of coordinates: the generalized function approach works only if the metric is $C^{1}$ for the step, and $C^{0}$ for the $\delta$-discontinuity. We transform, therefore, the Lagrangians and the Hamiltonians to the so-called surface form containing only smooth variables; such Lagrangians and Hamiltonians as well as symplectic forms decompose into sums of volume and surface integrals. The transformation can best be done in the so-called adapted coordinates; these are coordinates in which the embedding functions of the surfaces of discontinuity acquire the simplest possible form. The result, however, is covariant in the sense that arbitrary smooth coordinates can be chosen inside of each separated volume (left or right to the discontinuity surface) as well as along the discontinuity surface itself.

An important trick is used throughout the paper: we work in coordinate systems which are always adapted to the position of the discontinuity surface. This way the discontinuity surface may be considered as a fixed submanifold of the spacetime. Thus, the dynamics of the star surface or thin shell is not described by the spacetime coordinates of these objects but by the evolution of the physical fields like metric of matter fields along the surfaces. Then, for example the variations and time derivatives of the embedding functions of the two-surfaces of discontinuity in the three-surfaces of constant time both vanish identically. Our formulas are written only for one hypersurface of discontinuity; an extension to arbitrary many hypersurfaces is easy if they do not intersect each other.

Two interesting problems arise. First, we do not show that the dynamics makes sense even on-shell. By that, we mean that there is to be a well-posed initial value problem. One ought to be able to define some nice space of initial data, consisting of those values of the canonical variables that satisfy some well-defined set of constraints, jump and fall-off conditions so that a unique solution to the dynamical equations will exist in a neighbourhood of the initial surface. In this paper, we shall just assume that the dynamics is all right. At least in some special cases (like spherical symmetry), the space of classical solutions is well-known and it is as large as one expects.

The second problem is to show that the Hamiltonian formalism defines a (regular) constrained system. This means that one can find a phase space (possibly an extension of ours), a complete set of constraints, and a Hamiltonian satisfying the following conditions: 1) the constraints and Hamiltonian must be differentiable functions on the phase space so that their Poisson brackets are well-defined and 2) 
the Hamiltonian must be first class and the constraint set must be split nicely into the first and second class constraints (Bergmann-Dirac analysis, cf. [17]). Of course, such an 'off-shell' formulation is necessary as a starting point for Dirac quantization. The difficulty is that some constraints at the shell are not differentiable functions on the phase space even if they are smeared along the shell, because the smearing is then only two-dimensional, whereas the differentiability would require a threedimensional smearing. Without an off-sell formulation, the way to quantum theory need not be barred however. One can try to solve the singular constraints and to substitute the solution back into the action so that a variational principle results which leads to equivalent dynamics without the singular constraints [18]. A problem with such a procedure seems to be that equations quickly grow very messy.

The plan of the paper is as follows. Sec. 2 is devoted to the step, Sec. 3 to the $\delta$-function discontinuities. Sec. 2.1 introduces the ideal fluid model and its dynamics in a fixed spacetime (metric) background. Basic formulas of the Lagrange and Euler pictures concerning Lagrangians, Hamiltonians, strees-energy tensors and equations of motion are derived; these equations apply to both step and $\delta$ function discontinuity. The method of variation formulas is presented, which enables us to find the symplectic structure as well as to generate the equations of motion. The surface of discontinuity can be moved without problems as far as the metric is fixed. In Sec. 2.2, the fluid is coupled to the dynamical gravity. Relevant formulas concerning the variation of the Einstein-Hilbert action are collected. The surfaces of discontinuity are now fixed. This helps to avoid some formal problems. The variation formulas for the system are written in Lagrangian and Hamiltonian form.

In Sec. 3.1, an action for the thin shell and dynamical gravity is written down in the Lagrangian formalism; the shells are fixed and generalized functions are employed. In Sec. 3.2, the adapted coordinates are used to transform the (Lagrange formalism) action into a sum of volume and surface integrals disposing of the generalized functions and gaining more coordinate (gauge) freedom (four-covariance): arbitrary coordinates can be chosen left to the shell, right to the shell, and along the shell. In Sec. 3.3, the variation of the action in the surface form is calculated and the obtained dynamical equations are listed; they contain Israel's equation. The variation formula is derived; this is only three-covariant: the foliation by spacelike surfaces $t=$ const must be such that the $t$-surfaces are continuous but can have a cusp at the discontinuity surface; the embedding functions of the discontinuity twosurface in the $t$-surfaces must be time-independent. Sec. 3.4 contains a Legendre transformation to a Hamiltonian formalism; the general form of the Hamiltonian for the system of thin shells and gravity is presented. In sec. 3.5, the explicit functional dependence of the Hamiltonian on the dynamical variables is written down and the geometrical meaning of the surface terms in the Hamiltonian is disclosed. In Sec. 
3.6, the variation of the Hamiltonian is explicitly calculated so that all canonical equations and constraints following from the Hamiltonian can be listed. This not only enables us to check that the Hamiltonian generates the desired dynamics (including Israel's equation) but also to classify the resulting equations into 'canonical equations' and 'constraints.' For example, the six relations that are equivalent to Israel's equation consist of one super-Hamiltonian constraint, two supermomentum constraints, two singular constraints (these cannot be made differentiable by smearing), and one canonical equation. Some, necessarily preliminary, discussion of the result is given.

\section{Fluid with a step discontinuity}

Our point of departure in this section is the description of relativistic barotropic perfect fluid as given in Ref. [15] (observe that this description is easily extended to any conservative continuum). We will extend and modify the method so that it allows for discontinuous matter distributions admitting such situation like a jump of density at the boundary of a star (a step-function type of discontinuity along a timelike hypersurface).

\subsection{Fluid in gravitational field}

\subsubsection{The description of the fluid}

The fluid that have just 'mechanical' degrees of freedom consists of identifiable elementary volumes - mass points of the fluid. It can, therefore, be completely described by specifying the mass and the spacetime coordinates of each of these mass points. All mass points form the so-called matter space $Z$, which is a three dimensional manifold; let $z^{a}, a=1,2,3$, be some coordinates in $Z$. Let us denote the spacetime by $M$ and let $x^{\mu}, \mu=0,1,2,3$ be some coordinates in $M$. The state of the fluid can then be described by a map $\zeta: M \mapsto Z$, in coordinates $z^{a}\left(x^{\mu}\right)$; the particle trajectories are then determined by $z^{a}(x)=$ const. The matter space $Z$ is equipped with a scalar density $h(z)$, which determines the mole or particle density of the fluid, so that the number $\mathrm{N}$ of particles or moles in the volume $V_{z} \subset Z$ is given by $\mathrm{N}\left(V_{z}\right)=\int_{V_{z}} d^{3} z h$. We assume further that $h$ has a step discontinuity at a two surface $\Sigma_{z}$ in $Z$, defined by the equation $F(z)=0$, where $F$ is a smooth function with non-zero gradient $F_{a}$. Let $\Sigma:=\zeta^{-1}\left(\Sigma_{z}\right)$ be a timelike three-surface separating $M$ in two open subsets $V^{+}$and $V^{-}$so that $h(z(x))>0$ for $x \in V^{-}$and $h(z(x))=0$ for $x \in V^{+}$. One can make much more general assumptions (e.g. allowing for several matter filled regions), but this will only complicate the description without requiring any new method of approach. 
The map $\zeta$ and the density $h$ define mole (particle) current $j^{\mu}$ in $M$ by

$$
j^{\mu}=h \epsilon^{\mu \nu \kappa \lambda} z_{\nu}^{1} z_{\kappa}^{2} z_{\lambda}^{3},
$$

where

$$
z_{\mu}^{a}:=\frac{\partial z^{a}}{\partial x^{\mu}} .
$$

$j^{\mu}(x)$ is discontinuous at $\Sigma, j^{\mu} \neq 0$ in $V^{-}, j^{\mu}=0$ in $V^{+}$and $j_{-}^{\mu}$ is tangential to $\Sigma$. (We denote the limits to $\Sigma$ from inside by the index -.) $j^{\mu}$ is a vector density; it is easy to show that $j^{\mu}$ is identically conserved everywhere in $M, j^{\mu}{ }_{, \mu}=0$.

The current $j^{\mu}(x)$ defines the spacetime four-velocity $u^{\mu}(x)$ and the rest mole (particle) scalar density $n$ of the fluid in $V^{-}$and at $\Sigma$ by

$$
j^{\mu}=\sqrt{|g|} n u^{\mu},
$$

where $g:=\operatorname{det}\left(g_{\mu \nu}\right)$ and $g_{\mu \nu} u^{\mu} u^{\nu}=-1$. Hence,

$$
n=\frac{1}{\sqrt{|g|}} \sqrt{-g_{\mu \nu} j^{\mu} j^{\nu}}
$$

$n$ has a discontinuity of a step type at $\Sigma$.

In [15], it is shown that the fluid equations of motion can be obtained from the Lagrange density $L_{m}$ which is given by

$$
L_{m}=-\sqrt{|g|} n e(n),
$$

where $e(n)$ is the energy per mole in the rest frame of the fluid and $L_{m}$ is considered as a function of $z^{a}, z_{\mu}^{a}$ and $g_{\mu \nu}$. As the specific volume $V$ (i.e. volume of one mole in the rest frame) is $1 / n$, we obtain for the presure $p$ of the fluid

$$
p=-\frac{\partial e}{\partial V}=n^{2} e^{\prime}
$$

in $V^{-}$.

\subsubsection{Stress energy density}

By definition, the stress-energy tensor density of ideal fluid (see, eg. [9]) has the form

$$
T^{\mu \nu}=\sqrt{|g|}\left((\rho+p) u^{\mu} u^{\nu}+p g^{\mu \nu}\right),
$$

where

$$
\rho=n e(n)
$$

is the rest mass density; $T^{\mu \nu}$ has a step discontinuity at $\Sigma$. In this section, we collect some important formulas valid for this tensor density. 
Let us vary the action of the fluid

$$
I_{m}=-\int_{V^{-}} d^{4} x \sqrt{|g|} n e(n)
$$

with respect to $g_{\mu \nu}$. Using Eq. (3), we have

$$
\delta(\sqrt{|g|} n)=\delta \sqrt{-g_{\mu \nu} j^{\mu} j^{\nu}}=-\frac{1}{2 \sqrt{|g|}} n^{-1} j^{\mu} j^{\nu} \delta g_{\mu \nu},
$$

which, together with the Eq. (2) and the well-known variation formula for determinats yields

$$
\delta n=-\frac{1}{2} n\left(g^{\mu \nu}+u^{\mu} u^{\nu}\right) \delta g_{\mu \nu} .
$$

Then,

$$
\delta I_{m}=\int_{V^{-}} d^{4} x \sqrt{|g|}\left(-\frac{1}{2} g^{\mu \nu} n e+\frac{1}{2} n\left(g^{\mu \nu}+u^{\mu} u^{\nu}\right)\left(e+n e^{\prime}\right)\right) \delta g_{\mu \nu},
$$

and a straightforward calculation using Eqs. (7), (5) and (6) leads to

$$
T^{\mu \nu}(x)=2 \frac{\delta I_{m}}{\delta g_{\mu \nu}(x)} .
$$

The next important relation is the Belinfante-Rosenfeld theorem ([19], [20] and [21]) applied to our case: the Lagrange density $L_{m}$ must satisfy the following identity

$$
\frac{\partial L_{m}}{\partial z_{\mu}^{a}} z_{\nu}^{a}+2 \frac{\partial L_{m}}{\partial g_{\mu \rho}} g_{\nu \rho}=L_{m} \delta_{\nu}^{\mu} .
$$

This equation is equivalent to the requirement that $L_{m}$ is a scalar density, and its derivation is straightforward. From the identity (11) and the formula (10), we obtain immediately that

$$
T_{\nu}^{\mu}=L_{m} \delta_{\nu}^{\mu}-\frac{\partial L_{m}}{\partial z_{\mu}^{a}} z_{\nu}^{a} .
$$

Thus, the so called canonical stress-energy tensor density on the right hand side is equal to the source of gravitational field.

The formulas (10) and (12) imply the Noether identity:

$$
\nabla_{\mu} T_{\nu}^{\mu}=\left(-\partial_{\mu} \frac{\partial L_{m}}{\partial z_{\mu}^{a}}+\frac{\partial L_{m}}{\partial z^{a}}\right) z_{\nu}^{a} .
$$

There are counterparts to Eqs. (10)-(13) within any description of any type of ideal fluid. Derivation of Eq. (13) starts from the equation

$$
\nabla_{\mu} T_{\nu}^{\mu}=\partial_{\mu} T_{\nu}^{\mu}-\Gamma_{\mu \nu}^{\rho} T_{\rho}^{\mu} .
$$


If one substitutes for $T_{\nu}^{\mu}$ from Eq. (12) into the first term on the right hand side and from Eq. (10) into the second one, the identity follows. One consequence of the Noether identity is that the four components of the covector $\nabla_{\mu} T_{\nu}^{\mu}$ are not independent:

$$
j^{\nu} \nabla_{\mu} T_{\nu}^{\mu}=0,
$$

because the definition of $j^{\mu}$ implies the identity $j^{\mu} z_{\mu}^{a} \equiv 0$. Hence, the equation system $\nabla_{\mu} T_{\nu}^{\mu}=0$ contains only three independent equations (Euler equation); the energy conservation equation,

$$
\nabla_{\mu}\left(n e u^{\mu}\right)=-p \nabla_{\mu} u^{\mu},
$$

for the fluid is satisfied identically within our description.

\subsubsection{The variational formula}

Let us consider the 4-dimensional volume $V$ enclosed between two Cauchy surfaces $S_{1}$ and $S_{2}$; the boundary $\Sigma$ of the fluid divides $V$ into two parts, $V^{-}$and $V^{+}$, and similarly $S_{i}$ into $S_{i}^{-}$and $S_{i}^{+}, i=1,2$. We assume that $S_{1}$ and $S_{2}$ are $C^{1}$-surfaces, that is the induced metric on, as well as the unit normal vector to, $S_{1}$ and $S_{2}$ are both $C^{1}$.

Let us vary the matter action $I_{m}$ with respect to $z^{a}(x)$ and $g_{\mu \nu}$; we obtain

$$
\begin{aligned}
\delta I_{m} & =\int_{\delta V^{-}} d^{4} x L_{m}+\int_{V^{-}} d^{4} x \partial_{\mu}\left(\frac{\partial L_{m}}{\partial z_{\mu}^{a}} \delta z^{a}\right) \\
& +\int_{V^{-}} d^{4} x\left(\frac{\partial L_{m}}{\partial z^{a}}-\partial_{\mu} \frac{\partial L_{m}}{\partial z_{\mu}^{a}}\right) \delta z^{a}+\frac{1}{2} \int_{V^{-}} d^{4} x T^{\mu \nu} \delta g_{\mu \nu} .
\end{aligned}
$$

The first two integrals can be transformed to surface integrals along $\Sigma, S_{1}$ and $S_{2}$. For this aim, we use the coordinates $x^{\mu}$ in $M$ that are adapted to the surfaces. This means that $x^{0}=t_{i}$ along $S_{i}, i=1,2$ and

$$
F\left(z^{a}(x)\right)=x^{3}
$$

along $\Sigma$, so that $x^{k}, k=1,2,3$, are coordinates on $S_{i}$ and $x^{\alpha}, \alpha=0,1,2$ are coordinates on $\Sigma$. Then, the change of $F$ if we vary $z^{a}$ is given by

$$
F\left(z^{a}(x)+\delta z^{a}(x)\right)=x^{3}+F_{a} \delta z^{a},
$$

and the coordinate $x^{3}$ of $\Sigma$ changes by

$$
\delta x^{3}=-\left.F_{a} \delta z^{a}\right|_{x^{3}=0} .
$$


Thus, we obtain for the first term:

$$
\int_{\delta V^{-}} d^{4} x L_{m}=-\int_{\Sigma} d x^{0} d x^{1} d x^{2} L_{m} F_{a} \delta z^{a}
$$

For the second term, we have

$$
\int_{V^{-}} d^{4} x \partial_{\mu}\left(\frac{\partial L_{m}}{\partial z_{\mu}^{a}} \delta z^{a}\right)=\int_{\Sigma} d \Sigma \frac{\partial L_{m}}{\partial z_{3}^{a}} \delta z^{a}+\int_{S_{2}} d S \frac{\partial L_{m}}{\partial z_{0}^{a}} \delta z^{a}-\int_{S_{1}} d S \frac{\partial L_{m}}{\partial z_{0}^{a}} \delta z^{a}
$$

where the abbreviations $d \Sigma=d x^{0} d x^{1} d x^{2}$ and $d S=d x^{1} d x^{2} d x^{3}$ are used. Eq. (15) implies that $F_{a} z_{\mu}^{a}=\delta_{\mu}^{3}$, so we can write

$$
\frac{\partial L_{m}}{\partial z_{3}^{a}}=\frac{\partial L_{m}}{\partial z_{\mu}^{a}} F_{b} z_{\mu}^{b}
$$

Collecting these results, we obtain the equation

$$
\begin{aligned}
\delta I_{m} & =\int_{V^{-}} d^{4} x\left(\frac{\partial L_{m}}{\partial z^{a}}-\partial_{\mu} \frac{\partial L_{m}}{\partial z_{\mu}^{a}}\right) \delta z^{a}+\int_{\Sigma} d \Sigma\left(\frac{\partial L_{m}}{\partial z_{\mu}^{a}} z_{\mu}^{b}-L_{m} \delta_{a}^{b}\right) F_{b} \delta z^{a} \\
& +\int_{S_{2}} d S \frac{\partial L_{m}}{\partial z_{0}^{a}} \delta z^{a}-\int_{S_{1}} d S \frac{\partial L_{m}}{\partial z_{0}^{a}} \delta z^{a}+\frac{1}{2} \int_{V^{-}} d^{4} x T^{\mu \nu} \delta g_{\mu \nu}
\end{aligned}
$$

Thus, the field equations consist of volume equations that hold in $V^{-}$:

$$
\frac{\partial L_{m}}{\partial z^{a}}-\partial_{\mu} \frac{\partial L_{m}}{\partial z_{\mu}^{a}}=0
$$

and surface equations that hold at $\Sigma$ :

$$
\left(\frac{\partial L_{m}}{\partial z_{\mu}^{a}} z_{\mu}^{b}-L_{m} \delta_{a}^{b}\right) F_{b}=0
$$

The surface of the star is an observer independent dynamical element of the system.

Let us discuss the meaning of the field equations. For the volume equation (17), we just invoke Noether's identity, Eq. (13); we can then see that they are equivalent to the conservation equations $\nabla_{\mu} T_{\nu}^{\mu}=0$. The surface equations can be rewritten as follows. First, using Eq. (12), we have

$$
T_{\nu}^{\mu} z_{\mu}^{b}=\left(-\frac{\partial L_{m}}{\partial z_{\mu}^{a}} z_{\mu}^{b}+L_{m} \delta_{a}^{b}\right) z_{\nu}^{a}
$$

Hence,

$$
T_{\nu}^{\mu} F_{b} z_{\mu}^{b}=\left(-\frac{\partial L_{m}}{\partial z_{\mu}^{a}} z_{\mu}^{b}+L_{m} \delta_{a}^{b}\right) F_{b} z_{\nu}^{a}
$$


However, $F_{b} z_{\mu}^{b}$ is covector normal to $\Sigma$, so the three surface equations (18) can be written in a covariant form as

$$
\left.T_{\mu}^{\perp}\right|_{\Sigma}=0
$$

where $T_{\mu}^{\perp}=T_{\mu}^{\nu} \tilde{m}_{\nu}$ and $\tilde{m}_{\nu}$ is any normal covector to $\Sigma$. Of these four equations, only three are independent, because $T_{\nu}^{\perp} j^{\nu}$ is identically zero, as one easily verifies; in fact, the three equations $\left.T_{k}^{\perp}\right|_{\Sigma}=0$ imply (20). It follows further from Eq. (6) and from $F_{b} z_{\mu}^{b} u^{\mu}=0$ that Eq. (20) is equivalent to the condition that the pressure vanishes, $p=0$, at the surface.

Formula (16) is only valid in the adapted coordinates; in particular, the Lagrangian density $L_{m}$ must be expressed in these coordinates. Let us pass to more general coordinates. In fact, the volume integrals are already in a covariant form, so we have just to transform the boundary integrals. However, we will need explicitly only the integrals over the Cauchy surfaces.

Let us define

$$
p_{a}:=\frac{\partial L_{m}}{\partial z_{0}^{a}}
$$

The reader can easily verify that Eq. (21), which is written in the adapted coordinates, defines a three-density $p_{a}$ along $S_{i}$ (independent of the adapted coordinates), because the quantity $L_{m}$ is a four-density.

The field equations (both volume and surface) are, therefore, equivalent to the formula

$$
\delta \int_{V^{-}} d^{4} x L_{m}=\int_{S_{2}} d^{3} y p_{a} \delta z^{a}-\int_{S_{1}} d^{3} y p_{a} \delta z^{a}+\frac{1}{2} \int_{V^{-}} d^{4} x T^{\mu \nu} \delta g_{\mu \nu},
$$

and this formula is valid in any coordinates $x^{\mu}$ in $V^{-}$and $y^{k}, k=1,2,3$, along $S_{i}$. Let us denote the matter occupied part of the Cauchy surface $x^{0}=t$ by $S_{t}$ and the corresponding part of the matter space by $Z^{-}$. The following coordinates will simplify all calculations: the intersection $S_{t} \cap \Sigma$ is given by $x^{3}=x^{3}(t)$ and $S_{t}$ by $x^{3}<x^{3}(t) ; x^{k}, k=1,2,3$, are coordinates on $S_{t}$; we will call them 'time dependent adapted coordinates.' Then, we can write

$$
\delta \int_{V^{-}} d^{4} x L_{m}=\int_{t_{1}}^{t_{2}} d t\left(\frac{d}{d t} \int_{x^{3}<x^{3}(t)} d^{3} x p_{a} \delta z^{a}+\frac{1}{2} \int_{x^{3}<x^{3}(t)} d^{3} x T^{\mu \nu} \delta g_{\mu \nu}\right) .
$$

If we define the Lagragian $\mathcal{L}_{m}$ by

$$
\mathcal{L}_{m}=\int_{S_{t}} d^{3} x L_{m}
$$

and go to the limit $S_{2} \rightarrow S_{1}$, we obtain the variation formula for the Lagrangian:

$$
\delta \mathcal{L}_{m}=\int_{S_{t}} d^{3} x\left(p_{a} \delta z^{a}\right) \cdot \int_{\partial S_{t}} d^{2} x p_{a} \delta z^{a} \dot{x}^{3}+\frac{1}{2} \int_{S_{t}} d^{3} x T^{\mu \nu} \delta g_{\mu \nu}
$$


The variation formula has been derived by careful inclusion of all 'boundary terms'; this will be the main strategy for our derivation of the Lagrangian and Hamiltonian formalism that admit discontinuities. The role of the variational formula is to generate all dynamical equations (including the definition of momenta): the variation of the L. H. S. is to be calculated and compared with the R. H. S.

Eq. (22) is also the point of departure for the transformation to the Hamiltonian formalism.

\subsubsection{The Hamiltonian formalism}

Let us disregard the surface term in the formula (22) and define the Hamiltonian density $H_{m}$ by a Legendre transformation of the form

$$
H_{m}:=p_{a} \dot{z}^{a}-L_{m} .
$$

Then we obtain for the variation of the Hamiltonian $\mathcal{H}_{m}$, which is defined by

$$
\mathcal{H}_{m}:=\int_{S_{t}} d^{3} x H_{m}
$$

the relation

$$
\delta \mathcal{H}_{m}=\delta \int_{S_{t}} d^{3} x p_{a} \dot{z}^{a}-\delta \int_{S_{t}} d^{3} x L_{m}
$$

Performing carefully the variation in the first term and substituting from Eq. (22) for the second, we have

$$
\begin{aligned}
\delta \mathcal{H}_{m} & =\int_{S_{t}} d^{3} x\left(\delta p_{a} \dot{z}^{a}-\dot{p}_{a} \delta z^{a}\right)+\int_{x^{3}=x^{3}(t)} d^{2} x p_{a}\left(\dot{z}^{a} \delta x^{3}-\dot{x}^{3} \delta z^{a}\right) \\
& -\frac{1}{2} \int_{S_{t}} d^{3} x T^{\mu \nu} \delta g_{\mu \nu} .
\end{aligned}
$$

From this equation, not only the canonical equations are obtained, which will consist of volume and of surface equations, but also the symplectic structure of the system can be read off, which is given by the fist two integrals on the R.H.S.; these integals can be interpreted as $\omega\left(\delta z^{a}, \delta p_{a} ; \dot{z}^{a}, \dot{p}_{a}\right)$, where $\omega$ is the symplectic form and $\left(\delta z^{a}(x), \delta p_{a}(x)\right),\left(\dot{z}^{a}(x), \dot{p}_{a}(x)\right)$ are two vectors; notice that $\omega$ has a surface part. This is a general and very important observation, which will, for instance, help to decide what are the canonical variables for the shell in the second part of the paper. The last integral in (23) represents variation with respect to non-dynamical 'parameters' $g_{\mu \nu}(x)$.

We will need the following relations between the Hamiltonian density and the stress-energy density:

$$
\begin{aligned}
T_{0}^{0} & =-H_{m} \\
T_{k}^{0} & =-p_{a} z_{k}^{a} \\
T_{l}^{k} & =\left(\frac{\partial H_{m}}{\partial p_{a}} p_{a}-H_{m}\right) \delta_{l}^{k}+\frac{\partial H_{m}}{\partial z_{k}^{a}} z_{l}^{a}
\end{aligned}
$$


which are valid in the time dependent adapted coordinates. The first two equations are obtained immediately from Eq. (12) and the definition of $p_{a}$. To derive the last equation, we first notice that Eq. (23) has the following consequence

$$
T^{\mu \nu}=-2 \frac{\partial H_{m}}{\partial g_{\mu \nu}} .
$$

Second, we derive an equation analogous to (11) for $H_{m}$; we use the fact that $H_{m}$ behaves as a three-density if we change the coordinates $x^{k}$ keeping $t$ fixed and that $H_{m}$ is a function of $z^{a}, z_{m}^{a}, p_{a}$ and $g_{\mu \nu}, H_{m}=H_{m}\left(z^{a}, z_{\mu}^{a}, p_{a}, g_{\mu \nu}\right)$ :

$$
\frac{\partial H_{m}}{\partial z_{l}^{a}} z_{k}^{a}+\frac{\partial H_{m}}{\partial p_{a}} p_{a} \delta_{k}^{l}+2 \frac{\partial H_{m}}{\partial g_{\rho l}} g_{\rho k}=H_{m} \delta_{k}^{l} .
$$

Then, Eq. (26) follows immediately. Again, analogons of Eqs. (24)-(27) are valid for all types of ideal fluid.

To finish the Legendre transformation, we have also to express the velocity $\dot{z}^{a}$ in terms of $p_{a}, z^{a}$ and $z_{k}^{a}$ in the Hamiltonian. This is not completely straightforward. To begin with, we substitute for $L_{m}$ from Eq. (4) into the definition (21) of $p_{a}$ :

$$
p_{a}=-\sqrt{|g|} \rho^{\prime} \frac{\partial n}{\partial \dot{z}^{a}},
$$

where

$$
\rho^{\prime}=\frac{d \rho}{d n}
$$

Eqs. (2) and (3) imply

$$
\frac{\partial n}{\partial \dot{z}^{a}}=-\frac{1}{\sqrt{|g|}} u_{\mu} \frac{\partial j^{\mu}}{\partial \dot{z}^{a}}
$$

The definition of $j^{\mu}$ implies the following identity (cf. [15])

$$
\frac{\partial j^{\mu}}{\partial z_{\nu}^{a}} z_{\kappa}^{a}=j^{\mu} \delta_{\kappa}^{\nu}-j^{\nu} \delta_{\kappa}^{\mu}
$$

Combining the three equations, we easily find

$$
u_{k}=-\frac{p_{a} z_{k}^{a}}{\rho^{\prime} j^{0}}
$$

where $j^{0}$ depends only on $z^{a}$ and $z_{k}^{a}$ (cf. (11)):

$$
j^{0}=h(z) \operatorname{det}\left(z_{k}^{a}\right) .
$$

The (3+1)-decomposition of the metric (see e.g. [9]),

$$
\begin{array}{ll}
g^{00}=-N^{-2}, & g_{0 k}=N_{k}, \\
g_{k l}=q_{k l}, & g=-q N^{2},
\end{array}
$$


gives, with the help of Eq. (22),

$$
q^{k l} u_{k} u_{l}=-1-\frac{\left(u^{0}\right)^{2}}{g^{00}}=-1+\frac{1}{N^{2}}\left(\frac{j^{0}}{\sqrt{q}}\right)^{2} .
$$

Substituting for $u_{k}$ from Eq. (29), we obtain the identity

$$
\frac{1}{n^{2}}\left(\frac{j^{0}}{\sqrt{q}}\right)^{2}=1+\frac{q^{k l} z_{k}^{a} z_{l}^{b}}{\rho^{\prime 2}\left(j^{0}\right)^{2}} p_{a} p_{b}
$$

This equation determines $n$ as a function of $p_{a}, z^{a}$ and $z_{k}^{a}$. The solution depends on the unknown function $\rho(n)$ and is determined only implicitly, in general.

The identity (28) implies that

$$
\frac{\partial j^{k}}{\partial \dot{z}^{a}}=-j^{0} x_{a}^{k}
$$

where $x_{a}^{k}$ is the matrix inverse to $z_{k}^{a}$. As $j^{k}$ depends linearly on $\dot{z}^{a}$, we have

$$
j^{k}=-j^{0} x_{a}^{k} \dot{z}^{a}
$$

or

$$
\dot{z}^{a}=-\frac{z_{a}^{k} j^{k}}{j^{0}}
$$

Substituting for $j^{k}$ from

$$
j^{k}=g^{k l}\left(j_{l}-N_{l} j^{0}\right),
$$

for $j_{l}$ from (11) and for $u_{k}$ from (29), we obtain that

$$
\dot{z}^{a}=N \frac{\sqrt{q} n}{\rho^{\prime}\left(j^{0}\right)^{2}} q^{k l} z_{k}^{a} z_{l}^{b} p_{b}+N^{k} z_{k}^{a} .
$$

Then, Eqs. (12) and (21) yield

$$
T_{0}^{0}=-N \sqrt{q}\left(\rho+\frac{n}{\rho^{\prime}\left(j^{0}\right)^{2}} q^{k l} z_{k}^{a} z_{l}^{b} p_{a} p_{b}\right)-N^{k} z_{k}^{a} p_{a} .
$$

According to the formula (24), this determines the form of the Hamiltonian $\mathcal{H}_{m}$.

\subsubsection{The Euler picture}

At this stage, we have derived all important formulas of the Hamiltonian formalism in which the fluid is described by the functions $z^{a}(x)$; these are Lagrange coordinates (cf. [16]) and we can call the formalism 'Lagrange picture'. Sometimes the Euler 
picture is more practical, however. This can be obtained by the following canonical transformation. The new fields $x^{k}(z, t)$ (Euler coordinates) are defined by

$$
x^{k}(z(x, t), t)=x^{k}, \quad \forall x^{k}, t
$$

and the conjugate momenta $P_{k}$ by

$$
P_{k}(z, t):=-X(z, t) z_{k}^{a}(x(z, t), t) p_{a}(x(z, t), t),
$$

where

$$
X:=\operatorname{det}\left(\frac{\partial x^{k}}{\partial z^{a}}\right) .
$$

One easily checks that Eqs. (32) and (33) define a canonical transformation.

Let us first derive some useful relations. By differentiating Eq. (32) with respect to $t$ at constant $x^{k}$, we obtain

$$
\dot{x}^{k}=-x_{a}^{k} \dot{z}^{a}
$$

where

$$
x_{a}^{k}:=\frac{\partial x^{k}}{\partial z^{a}} .
$$

The derivative of the same equation with respect to $x^{k}$ at constant $t$ gives

$$
x_{a}^{k} z_{l}^{a}=\delta_{l}^{k} .
$$

If the field $z^{a}(x, t)$ is changed to $\tilde{z}^{a}(x, t)$, then $x^{k}(z, t)$ is changed to $\tilde{x}^{k}(z, t)$ satisfying

$$
\tilde{x}^{k}(\tilde{z}(x, t), t)=x^{k}, \quad \forall x^{k}, t .
$$

Thus, if $\tilde{z}^{a}(x, t)=z^{a}(x, t)+\delta z^{a}(x, t)$, the above equation implies that

$$
\delta_{*} x^{k}=-x_{a}^{k} \delta z^{a} .
$$

The symbol $\delta_{*}$ is to stress and to remind us that this variation is of different kind than $\delta$, if applied to fields: the former is obtained by comparing the values of the field at the same point of the matter space, that is at different points of the spacetime; the latter compares the values of the field at the same point of the spacetime.

With the help of the above relations, we can transform all formulas of the Hamiltonian formalism. Let us start with Eq. (23). First, the inverse transformation for the momenta follows from Eq. (33):

$$
p_{a}(x, t)=-X^{-1}(x, t) x_{a}^{k}(z(x, t), t) P_{k}(z(x, t) t),
$$

The time derivative of this equation at constant $x^{k}$ can be calculated with the result

$$
\begin{aligned}
\dot{p}_{a} & =X^{-1}\left(x_{a}^{k} x_{b}^{m} z_{l m}^{b} P_{k} \dot{x}^{l}+z_{l}^{b} x_{a b}^{k} P_{k} \dot{x}^{l}+x_{a}^{k} z_{l}^{b} P_{k} \partial_{b} \dot{x}^{l}+x_{a}^{k} z_{l}^{b} \partial_{b} P_{k} \dot{x}^{l}\right. \\
& \left.-P_{l} \partial_{a} \dot{x}^{l}-x_{a}^{k} \dot{P}_{k}\right)
\end{aligned}
$$


where we introduced the abbreviation

$$
z_{k l}^{a}:=\frac{\partial^{2} z^{a}}{\partial x^{k} \partial x^{l}}
$$

and similarly $x_{a b}^{k}$. Analogous formula holds for the variation $\delta p_{a}$, one just have to replace dots by $\delta$ 's. Employing these equations, we obtain after a lenghty but straightforward calculation

$\delta p_{a} \dot{z}^{a}-\dot{p}_{a} \delta z^{a}=X^{-1}\left(\delta_{*} P_{k} \dot{x}^{k}-\dot{P}_{k} \delta_{*} x^{k}\right)+X^{-1} \partial_{a}\left(P_{k} z_{l}^{a} \delta_{*} x^{k} \dot{x}^{l}\right)-X^{-1} \partial_{a}\left(P_{l} z_{k}^{a} \delta_{*} x^{k} \dot{x}^{l}\right)$,

or

$$
\begin{aligned}
\int_{S_{t}} d^{3} x\left(\delta p_{a} \dot{z}^{a}-\dot{p}_{a} \delta z^{a}\right) & =\int_{Z^{-}} d^{3} z\left(\delta_{*} P_{k} \dot{x}^{k}-\dot{P}_{k} \delta_{*} x^{k}\right) \\
& +\int_{Z^{-}} d^{3} z \partial_{a}\left(\left(P_{k} z_{l}^{a}-P_{l} z_{k}^{a}\right) \delta_{*} x^{k} \dot{x}^{l}\right) .
\end{aligned}
$$

Then, we transform the second integral on the R.H.S. of Eq. (23):

$$
\begin{aligned}
\int_{x^{3}=x^{3}(t)} d^{2} x p_{a}\left(\dot{z}^{a} \delta x^{3}-\dot{x}^{3} \delta z^{a}\right) & =\int_{S_{t}} d^{3} x \partial_{l}\left(p_{a}\left(\dot{z}^{a} \delta_{*} x^{l}-\dot{x}^{l} \delta z^{a}\right)\right) \\
& =\int_{Z^{-}} d^{3} z X z_{l}^{b} \partial_{b}\left(X^{-1} P_{k}\left(\dot{x}^{k} \delta_{*} x^{l}-\dot{x}^{l} \delta_{*} x^{k}\right)\right)
\end{aligned}
$$

we have used Eqs. (34), (36),(35) and (37). Because of the identity $\partial_{b}\left(X z_{l}^{b}\right)=0$, we obtain finally

$$
\int_{x^{3}=x^{3}(t)} d^{2} x p_{a}\left(\dot{z}^{a} \delta x^{3}-\dot{x}^{3} \delta z^{a}\right)=-\int_{Z^{-}} d^{3} z \partial_{b}\left(\left(P_{k} z_{l}^{b}-P_{l} z_{k}^{b}\right) \delta_{*} x^{k} \dot{x}^{l}\right) .
$$

Eqs. (23), (39) and (40) imply

$$
\delta \mathcal{H}_{m}=\int_{Z^{-}} d^{3} z\left(\delta_{*} P_{k} \dot{x}^{k}-\dot{P}_{k} \delta_{*} x^{k}\right)+\frac{1}{2} \int_{Z^{-}} d^{3} z X T^{\mu \nu} \delta g_{\mu \nu}(x(x, t), t) .
$$

Thus, the symplectic form has no surface term in the Euler picture. The variation of the metric in the last term on the R.H.S. is independent of the other variations, and it is defined by comparing values of the metric at the same spacetime points.

Let us suppose that $\delta g_{\mu \nu}(x)=0$, and let us introduce the transformed Hamiltonian density $H_{m}^{\prime}$ by $H_{m}^{\prime}=X H_{m}$, so that

$$
\mathcal{H}_{m}=\int_{Z^{-}} d^{3} z H_{m}^{\prime}
$$

Then,

$$
\delta \mathcal{H}_{m}=\int_{Z^{-}} d^{3} z \delta_{*} H_{m}^{\prime}
$$


and we have

$$
\int_{Z^{-}} d^{3} z \delta_{*} H_{m}^{\prime}=\int_{Z^{-}} d^{3} z\left(\delta_{*} P_{k} \dot{x}^{k}-\dot{P}_{k} \delta_{*} x^{k}\right)
$$

In this form, the variational formula is suitable for derivation of the canonical equations. To this aim, let us calculate $\delta_{*} H_{m}^{\prime} ; H_{m}^{\prime}$ is of the form $H_{m}^{\prime}\left(x^{k}, x_{a}^{k}, P_{k}\right)$, hence

$$
\delta_{*} H_{m}^{\prime}=\left(\frac{\partial H_{m}^{\prime}}{\partial x^{k}}-\partial_{a} \frac{\partial H_{m}^{\prime}}{\partial x_{a}^{k}}\right) \delta_{*} x^{k}+\frac{\partial H_{m}^{\prime}}{\partial P_{k}} \delta_{*} P_{k}+\partial_{a}\left(\frac{\partial H_{m}^{\prime}}{\partial x_{a}^{k}} \delta_{*} x^{k}\right) .
$$

Thus, the field equations consist of the volume equations:

$$
\begin{aligned}
\dot{x}^{k} & =\frac{\partial H_{m}^{\prime}}{\partial P_{k}} \\
-\dot{P}_{k} & =\frac{\partial H_{m}^{\prime}}{\partial x^{k}}-\partial_{a} \frac{\partial H_{m}^{\prime}}{\partial x_{a}^{k}}
\end{aligned}
$$

and the surface equations:

$$
\left.F_{a} \frac{\partial H_{m}^{\prime}}{\partial x_{a}^{k}}\right|_{\Sigma_{z}}=0
$$

Let us check that Eq. (45) is equivalent to (20). We have

$$
H_{m}^{\prime}=X H_{m}\left(z^{a}\left(x^{l}\right), z_{k}^{a}\left(x_{b}^{l}\right), p_{a}\left(z^{a}\left(x^{l}\right), z_{k}^{a}\left(x_{b}^{l}\right), P_{k}\right), g_{\mu \nu}\left(x^{l}\right)\right)
$$

so that

$$
\left.\frac{\partial H_{m}^{\prime}}{\partial x_{a}^{k}}\right|_{x, P}=X z_{k}^{a} H_{m}^{\prime}+\left.X \frac{\partial H_{m}^{\prime}}{\partial z_{l}^{b}}\right|_{z, p} \frac{\partial z_{l}^{b}}{\partial x_{a}^{k}}+\left.X \frac{\partial H_{m}^{\prime}}{\partial p_{b}}\right|_{z, z_{k}} \frac{\partial p_{b}}{\partial x_{a}^{k}} .
$$

Eqs. (35) and (37) imply

$$
\begin{aligned}
& \frac{\partial z_{l}^{b}}{\partial x_{a}^{k}}=-z_{k}^{b} z_{l}^{a} \\
& \frac{\partial p_{b}}{\partial x_{a}^{k}}=-p_{b} z_{k}^{a}+\delta_{b}^{a} p_{c} z_{k}^{c}
\end{aligned}
$$

and we obtain easily

$$
\frac{\partial H_{m}^{\prime}}{\partial x_{a}^{k}}=X z_{l}^{a}\left(\left(H_{m}-\frac{\partial H_{m}}{\partial p_{b}} p_{b}\right) \delta_{k}^{l}-\frac{\partial H_{m}}{\partial z_{l}^{b}} z_{k}^{b}\right)+X \frac{\partial H_{m}}{\partial p_{a}} p_{b} z_{k}^{b} .
$$

Application of Eqs. (25) and (26) as well as (23) simplify the expression to

$$
\frac{\partial H_{m}^{\prime}}{\partial x_{a}^{k}}=-X z_{\mu}^{a} T_{k}^{\mu}
$$

Hence, Eq. (45) becomes

$$
\left.F_{a} z_{\mu}^{a} T_{k}^{\mu}\right|_{\Sigma_{z}}=0
$$


which is equivalent to (20).

Finally, the transformed Eq. (30) reads

$$
n^{2}+\frac{n^{2}}{h^{2} \rho^{\prime 2}} q^{k l} P_{k} P_{l}=\frac{h^{2}}{q X^{2}} .
$$

For example, in the case of dust, $\rho=\mu n$, where $\mu$ is a constant (rest mass per mole or particle) and Eq. (47) can be solved explicitly:

$$
n=\frac{1}{\sqrt{q} X} \frac{\mu h^{2}}{\sqrt{\mu^{2} h^{2}+q^{k l} P_{k} P_{l}}} .
$$

For dust, Eq. (31), which determines the form of the matter Hamiltonian, specializes to

$$
T_{0}^{0}=-N \sqrt{\mu^{2}\left(j^{0}\right)^{2}+q^{k l} z_{k}^{a} z_{l}^{b} p_{a} p_{b}}-N^{k} z_{k}^{a} p_{a} .
$$

\subsection{The gravity becomes dynamical}

In the foregoing sections, gravity was just an external field. Here, it will become dynamical: the metric $g_{\mu \nu}(x)$ will satisfy Einstein's equations with the fluid stress energy tensor as a source.

\subsubsection{Description of the system}

The main problem which we shall meet is the following. If Einstein's equations are satisfied, the discontinuity in the distribution of the fluid leads to a discontinuity in derivatives of the metric. Thus, we must allow for such discontinuity from the very beginning. Moreover, a general variation of the metric, which includes a shift of the coordinates of the discontinuity, will have a jump of higher order than the metric itself: if the second derivatives of the metric have a jump, then the first derivative of its variation will have a jump, etc. If we write naively the usual expression for the variation of the action in the case of delta-function fluid distribution, then many terms in it look meaningless within the theory of distributions ( $\delta$-functions multiplied by discontinuous functions, etc.). Some ingenious calculation of all variations might still lead to meaningful expressions. Instead, we resort to a simple trick by which the problem is avoided: we fix the spacetime coordinates of the discontinuity surface $\Sigma$. In this way, the surface of the discontinuity is formally made to an 'externally given' boundary. The fields $z^{a}(x)$ and $g_{\mu \nu}(x)$ will satisfy simple boundary conditions at $\Sigma$, and these conditions will be 'inherited' by their variations. Such a strategy is possible within the general relativity, because it can be considered as a partial fixing of gauge. Indeed, any change of the coordinates of the discontinuity surface $\Sigma$ can be considered as a superposition of a transformation of coordinates in a neighbourhood 
of $\Sigma$ keeping the physical fields fixed, and a change of the physical fields keeping the coordinates fixed; the first step is just a change of gauge. The dynamics of the surface is determined by the form of the metric near and at the surface.

To be more specific about the boundary conditions, let us choose the coordinates $z^{a}$ in $Z$ such that $\Sigma_{z}$ is given by $z^{3}=0$, and the coordinates $x^{\mu}$ in $M$ such that $\Sigma$ is defined by $x^{3}=0$. Thus, for the matter fields, we require

$$
\left.z^{3}\right|_{\Sigma}=0,\left.\quad \delta z^{3}\right|_{\Sigma}=0
$$

It follows that

$$
\left.x^{3}\right|_{\Sigma_{z}}=0,\left.\quad \delta x^{3}\right|_{\Sigma_{z}}=0
$$

in the Euler picture. We further assume that

Condition 1 the spacetime $(M, g)$ is asymptotically flat and globally hyperbolic;

Condition 2 the metric $g_{\mu \nu}(x)$ is piecewise $C^{\infty}$ in $M$, the only discontinuity being that its second derivatives jump at $\Sigma$.

Then the variation $\delta g_{\mu \nu}(x)$ satisfies analogous Condition 2 .

The total action for our fluid-gravity system is $I=\bar{I}_{m}+I_{g}$. Here

$$
I_{g}=\frac{1}{16 \pi \mathrm{G}} \int_{V} d^{4} x \sqrt{|g|} R
$$

where $\mathrm{G}$ is the Newton constant and $R$ is the curvature scalar of $g_{\mu \nu}$. The function $R(x)$ can have a step discontinuity at $\Sigma . \bar{I}_{m}$ is obtained from $I_{m}$ of Eq. (8) after the following substitution

$$
z^{3}\left(x^{\alpha}, x^{3}=0\right)=0, \quad \dot{z}^{3}\left(x^{\alpha}, x^{3}=0\right)=0, \quad z_{A}^{3}\left(x^{\alpha}, x^{3}=0\right)=0,
$$

$\alpha=0,1,2$ and $A=1,2$. Thus, $\bar{I}_{m}$ contains less variables than $I_{m}$.

The integration volume $V$ is chosen to be bounded by two Cauchy surfaces, $S_{1}$ and $S_{2}$, and by a timelike surface $\Sigma^{+}$(which will be eventually pushed to the infinity). Let the coordinates $x^{\mu}$ be adapted also to $\Sigma^{+}$so that $\Sigma^{+}$is defined by $x^{3}=r^{+}$. The matter boundary $\Sigma$ divides $V$ into $V^{-}$and $V^{+}$, and $S_{i}$ into $S_{i}^{-}$and $S_{i}^{+}$.

\subsubsection{The variational formula}

The variation of the gravity action $I_{g}$ can be obtained from the following fundamental lemma that has been shown in [14]. 
Lemma 1 Let the integration volume $V$ of the action $I_{g}$ be bounded by two spacelike surfaces $S_{1}$ and $S_{2}$, and by a smooth timelike surface $\Sigma$; let $x^{\mu}$ be some coordinates in $V, y^{k}$ in $S_{i}, \xi^{\alpha}$ in $\Sigma$ and $\eta^{A}$ in $\partial S_{i}=\Sigma \cap S_{i}$. Then

$$
\begin{aligned}
\delta I_{g} & =-\frac{1}{16 \pi G} \int_{V} d^{4} x G^{\mu \nu} \delta g_{\mu \nu} \\
& -\frac{1}{16 \pi G} \int_{S_{2}} d^{3} y q_{k l} \delta \pi^{k l}+\frac{1}{16 \pi G} \int_{S_{1}} d^{3} y q_{k l} \delta \pi^{k l} \\
& +\frac{1}{8 \pi G} \int_{\partial S_{2}} d^{2} \eta \sqrt{\lambda} \delta \alpha-\frac{1}{8 \pi G} \int_{\partial S_{1}} d^{2} \eta \sqrt{\lambda} \delta \alpha \\
& -\frac{1}{16 \pi G} \int_{\Sigma} d^{3} \xi \gamma_{\alpha \beta} \delta Q^{\alpha \beta}
\end{aligned}
$$

where

$$
G^{\mu \nu}:=\sqrt{|g|}\left(R^{\mu \nu}-\frac{1}{2} g^{\mu \nu} R\right)
$$

$R^{\mu \nu}$ is the Ricci tensor of the metric $g_{\mu \nu}, q_{k l}$ is the induced metric on $S_{i}$ written with respect to the coordinates $y^{k}, q$ its determinant,

$$
\begin{aligned}
\pi^{k l} & :=\sqrt{q}\left(K q^{k l}-K^{k l}\right) \\
K_{k l} & =-n_{\mu ; \nu} \frac{\partial x^{\mu}}{\partial y^{k}} \frac{\partial x^{\nu}}{\partial y^{l}} \\
K & =q^{k l} K_{k l}
\end{aligned}
$$

$n_{\mu}$ is the future directed unit normal to $S_{i}$ so that $K_{k l}$ is the second fundamental form of the surface $S_{i}, \gamma_{\alpha \beta}$ is the metric induced on $\Sigma$ written with respect to the coordinates $\xi^{\alpha}, \gamma$ its determinant

$$
\begin{aligned}
Q^{\alpha \beta} & :=\sqrt{|\gamma|}\left(L \gamma^{\alpha \beta}-L^{\alpha \beta}\right) \\
L_{\alpha \beta} & =\tilde{m}_{\mu ; \nu} \frac{\partial x^{\mu}}{\partial \xi^{\alpha}} \frac{\partial x^{\mu}}{\partial \xi^{\beta}} \\
L & =\gamma^{\alpha \beta} L_{\alpha \beta}
\end{aligned}
$$

$\tilde{m}_{\mu}$ is the external (with respect to the volume $V$ ) unit normal to $\Sigma$ so that $L_{\alpha \beta}$ is the second fundamental form of $\Sigma, \lambda$ is the determinant of the 2-metric $\lambda_{A B}$ induced on $\partial S_{i}$ written with respect to the coordinates $\eta^{A}$ and $\alpha$ is defined by

$$
\alpha:=-\operatorname{arcsinh}\left(g_{\mu \nu} n^{\mu} \tilde{m}^{\nu}\right)
$$

The Lemma 1 is completely general, independent of the form and description of the matter; it determines the 'gravitational part' of the variation formula that we are going to derive. For the 'matter part', we can use the formula (16) in which the 
surface integral along $\Sigma$ is left out. Indeed, $F(z)=z^{3}$ for our special coordinates and the boundary condition (49) gives $F_{a} \delta z^{a}=0$. Hence,

$$
\begin{aligned}
\delta I_{m} & =\int_{V^{-}} d^{4} x\left(\frac{\partial \bar{L}_{m}}{\partial z^{a}}-\partial_{\mu} \frac{\partial \bar{L}_{m}}{\partial z_{\mu}^{a}}\right) \delta z^{a}+\int_{S_{2}^{-}} d S \frac{\partial \bar{L}_{m}}{\partial z_{0}^{a}} \delta z^{a}-\int_{S_{1}^{-}} d S \frac{\partial \bar{L}_{m}}{\partial z_{0}^{a}} \delta z^{a} \\
& +\frac{1}{2} \int_{V^{-}} d^{4} x T^{\mu \nu} \delta g_{\mu \nu} .
\end{aligned}
$$

Our next task is to rewrite the surface integrals in Eq. (55) in a covariant way. We define, in analogy with Eq. (21),

$$
\bar{p}_{a}:=\frac{\partial \bar{L}_{m}}{\partial \dot{z}^{a}}
$$

By a similar argument as in Sec. 2.1.3, $\bar{p}_{a}$ are surface densities, and the covariant forn of the integrals is:

$$
\int_{S_{2}^{-}} d S \frac{\partial \bar{L}_{m}}{\partial z_{0}^{a}} \delta z^{a}-\int_{S_{1}^{-}} d S \frac{\partial \bar{L}_{m}}{\partial z_{0}^{a}} \delta z^{a}=\int_{S_{2}^{-}} d^{3} y \bar{p}_{a} \delta z^{a}-\int_{S_{1}^{-}} d^{3} y \bar{p}_{a} \delta z^{a} .
$$

The relations between the old and new matter momenta will play some role. They can be summarized as follows: in $V^{-}$, we simply have

$$
\bar{p}_{a}=p_{a}
$$

whereas at $\Sigma$,

$$
\bar{p}_{A}=p_{A}, \quad p_{3}=p\left(\bar{p}_{A}, z^{a}, z_{k}^{a}\right),
$$

where $p$ is some function of the variables indicated. Eq. (57) and the first Eq. of (58) follow directly from the definitions, if the substitutions (51) is made in the expressions on the R.H.S.'s. As $\bar{L}_{m}$ does not depend on $\dot{z}^{3}$ at the boundary, there is no $\left.\bar{p}_{3}\right|_{\Sigma} ;\left.p_{3}\right|_{\Sigma}$ as given by Eq. (21) with the substitutions (51) is, however, non-zero and it can be expressed as in the second Eq. of (58). Let us give a proof. The solution of Eq. (21) with respect to $\dot{z}^{a}$ reads

$$
\dot{z}^{a}=\dot{z}^{a}\left(p_{a}, z^{a}, z_{k}^{a}\right)
$$

At $\Sigma$, we must have $\dot{z}^{3}=0$, so we obtain one constraint for the functions $\left.p_{a}\right|_{\Sigma}$ :

$$
\dot{z}^{3}\left(p_{a}, z^{a}, z_{k}^{a}\right)=0
$$

This can be solved for $\left.p_{3}\right|_{\Sigma}$; the second Eq. of (58) is the solution.

As an example, we work out the explicit form of Eq. (59) for the dust. We easily obtain from Eq. (11)

$$
j^{k}=-h X^{-1} x_{a}^{k} \dot{z}^{a}
$$


if we observe that the intermediately resulting terms can be expressed by means of $z_{k}^{a}$-derivatives of the determinant of the matrix $z_{k}^{a}$. Eq. ([3) yields

$$
n=\frac{h}{X \sqrt{|g|}} \sqrt{-g_{00}+2 \tilde{N}_{a} \dot{z}^{a}-\tilde{q}_{a b} \dot{z}^{a} \dot{z}^{b}},
$$

where

$$
\begin{aligned}
\tilde{N}_{a} & :=N_{k} x_{a}^{k}, \\
\tilde{q}_{a b} & :=g_{k l} x_{a}^{k} x_{b}^{l} .
\end{aligned}
$$

Then, for $\rho=\mu n$, we have from (21) and (四):

$$
p_{a}=-\mu \frac{h^{2}}{X^{2} \sqrt{|g|}} \frac{1}{n}\left(\tilde{N}_{a}-\tilde{q}_{a b} \dot{z}^{b}\right),
$$

so that

$$
\dot{z}^{a}=\tilde{q}^{a b}\left(\tilde{N}_{b}+\frac{X^{2} \sqrt{|g|}}{\mu h^{2}} n p_{b}\right),
$$

and the desired constraint (59) reads

$$
\tilde{N}^{3}+n \frac{X^{2} N \sqrt{q}}{\mu h^{2}} \tilde{q}^{3 a} p_{a}=0,
$$

where

$$
\tilde{q}^{a b}:=q^{k l} z_{k}^{a} z_{l}^{b}
$$

and

$$
\tilde{N}^{a}:=N^{k} z_{k}^{a} .
$$

For $n$, we have to insert from Eq. (30):

$$
\frac{1}{n}=\frac{X^{2} \sqrt{q}}{\mu h^{2}} \sqrt{\mu^{2} h^{2} X^{-2}+\tilde{q}^{a b} p_{a} p_{b}} .
$$

Thus, the constraint (60) can be written as a quadratic equation for $p_{3}$, whose general solution is

$$
p_{3 \pm}=-\frac{\tilde{q}^{3 A}}{\tilde{q}^{33}} p_{A} \pm \tilde{N}^{3} \sqrt{\frac{\tilde{\lambda}^{A B} p_{A} p_{B}+\mu^{2} h^{2} X^{-2}}{\tilde{q}^{33}\left(N^{2} \tilde{q}^{33}-\left(\tilde{N}^{3}\right)^{2}\right)}}
$$

where

$$
\tilde{\lambda}^{A B}=\lambda^{C D} z_{C}^{A} z_{D}^{B}
$$


and

$$
\lambda_{A B}=q_{A B}
$$

is the metric induced on $\partial S$. Only the lower sign is admissible, as we can easily see from Eq. (60). Hence, finally,

$$
\left.p_{3}\right|_{\Sigma}=-\frac{\tilde{q}^{3 A}}{\tilde{q}^{33}} p_{A}-\tilde{N}^{3} \sqrt{\frac{\tilde{\lambda}^{A B} p_{A} p_{B}+\mu^{2} h^{2} X^{-2}}{\tilde{q}^{33}\left(N^{2} \tilde{q}^{33}-\left(\tilde{N}^{3}\right)^{2}\right)}} .
$$

Eqs. (52), (55) and (56) imply the final variational formula for our gravity-fluid system:

$$
\begin{aligned}
\delta I & =\int_{V^{-}} d^{4} x\left(\frac{\partial \bar{L}_{m}}{\partial z^{a}}-\partial_{\mu} \frac{\partial \bar{L}_{m}}{\partial z_{\mu}^{a}}\right) \delta z^{a}-\frac{1}{16 \pi \mathrm{G}} \int_{V} d^{4} x G^{\mu \nu} \delta g_{\mu \nu} \\
& +\int_{S_{2}^{-}} d^{3} y \bar{p}_{a} \delta z^{a}-\int_{S_{1}^{-}} d^{3} y \bar{p}_{a} \delta z^{a} \\
& -\frac{1}{16 \pi \mathrm{G}} \int_{S_{2}} d^{3} y q_{k l} \delta \pi^{k l}+\frac{1}{16 \pi \mathrm{G}} \int_{S_{1}} d^{3} y q_{k l} \delta \pi^{k l} \\
& +\frac{1}{8 \pi \mathrm{G}} \int_{\partial S_{2}} d^{2} \eta \sqrt{\lambda} \delta \alpha-\frac{1}{8 \pi \mathrm{G}} \int_{\partial S_{1}} d^{2} \eta \sqrt{\lambda} \delta \alpha \\
& -\frac{1}{16 \pi \mathrm{G}} \int_{\Sigma} d^{3} \xi \gamma_{\alpha \beta} \delta Q^{\alpha \beta}+\frac{1}{2} \int_{V^{-}} d^{4} x T^{\mu \nu} \delta g_{\mu \nu} .
\end{aligned}
$$

From the formula (61) we can read off the field equations; within $V^{-}$, we have:

$$
\begin{aligned}
\frac{\partial L_{m}}{\partial z^{a}}-\partial_{\mu} \frac{\partial L_{m}}{\partial z_{\mu}^{a}} & =0 \\
G^{\mu \nu} & =8 \pi G T^{\mu \nu}
\end{aligned}
$$

and within $V^{+}$, we have:

$$
G^{\mu \nu}=0
$$

Apparently, the surface equation (18) has been lost. However, using the boundary Condition 2, we easily find that $G_{\mu}^{\perp}$ is continuous at $\Sigma$. Hence,

$$
\lim _{x^{3}=0-} G_{\mu}^{\perp}=0
$$

and the surface field equation follows from Eq. (63). We also observe that the dynamics can be completely shifted to the gravity if the ideal fluid is described by co-moving coordinates everywhere in $V^{-}$.

Putting everything together, we obtain in an analogous way as in Sec. 2.1.3: the field equations are equivalent to the following relation (which is an analogon of Eq. 
(5.16) of [14])

$$
\begin{aligned}
\delta \mathcal{L} & =\int_{S^{-}} d^{3} y\left(\bar{p}_{a} \delta z^{a}\right)^{\cdot}-\frac{1}{16 \pi \mathrm{G}} \int_{S} d^{3} y\left(q_{k l} \delta \pi^{k l}\right)^{\cdot}+\frac{1}{8 \pi \mathrm{G}} \int_{\partial S} d^{2} \eta(\sqrt{\lambda} \delta \alpha)^{.} \\
& -\frac{1}{16 \pi \mathrm{G}} \int_{\partial S} d^{2} \eta \gamma_{\alpha \beta} \delta Q^{\alpha \beta},
\end{aligned}
$$

where $\mathcal{L}$ is the Lagrangian of the system,

$$
\mathcal{L}:=\int_{S} d S \bar{L} .
$$

Eq. (65) is the variation formula for our system.

\subsubsection{The Hamiltonian formalism}

Eq. (65) is a good starting point for the Legendre transformation to a Hamiltonian formalism. We define one of the conceivable total Hamiltonians for our system by

$$
\check{\mathcal{H}}:=\int_{S^{-}} d^{3} y \bar{p}_{a} \dot{z}^{a}-\frac{1}{16 \pi \mathrm{G}} \int_{S} d^{3} y q_{k l} \dot{\pi}^{k l}+\frac{1}{8 \pi \mathrm{G}} \int_{\partial S} d^{2} \eta \sqrt{\lambda} \dot{\alpha}-\mathcal{L} .
$$

Then, the field equations can be obtained from the variation formula

$$
\begin{aligned}
\delta \check{\mathcal{H}} & =\int_{S^{-}} d^{3} y\left(\dot{z}^{a} \delta \bar{p}_{a}-\dot{\bar{p}}_{a} \delta z^{a}\right)+\frac{1}{16 \pi \mathrm{G}} \int_{S} d^{3} y\left(\dot{q}_{k l} \delta \pi^{k l}-\dot{\pi}^{k l} \delta q_{k l}\right) \\
& +\frac{1}{8 \pi \mathrm{G}} \int_{\partial S} d^{2} \eta \frac{1}{2 \sqrt{\lambda}}(\dot{\alpha} \delta \lambda-\dot{\lambda} \delta \alpha)+\frac{1}{16 \pi \mathrm{G}} \int_{\partial S} d^{2} \eta \gamma_{\alpha \beta} \delta Q^{\alpha \beta} .
\end{aligned}
$$

To find the explicit form of the Hamiltonian we use the lemma

Lemma 2 In the adapted coordinates $x^{\mu}$ defined in Sec. 2.2.1, the following identity holds at any $S=S_{t}$ in the volume $V$

$$
\begin{aligned}
& \int_{S} d S q_{k l} \dot{\pi}^{k l}-2 \int_{\partial S} d \partial S \sqrt{\lambda} \dot{\alpha}= \\
& \quad-2 \int_{S} d S \sqrt{|g|} R_{0}^{0}-2 \int_{\partial S} d \partial S \sqrt{|\gamma|} L_{0}^{0},
\end{aligned}
$$

where $L^{\alpha \beta}$ is the second fundamental form of the boundary $\partial V$ corresponding to the normal oriented outwards from $V, d S=d x^{1} d x^{2} d x^{3}$ and $d \partial S=d x^{1} d x^{2}$.

The derivation of this identity is given in 14] (Eq. (6.3)); the form (68) is easily obtained if one uses the equation $Q^{A B} g_{A B}-Q^{00} g_{00}=\sqrt{|\gamma|} L_{0}^{0}$, which follows from Eq. (54). We also observe that

$$
\int_{S^{-}} d S \bar{p}_{a} \dot{z}^{a}-\overline{\mathcal{L}}_{m}=\overline{\mathcal{H}}_{m}
$$


where $\overline{\mathcal{H}}_{m}$ is obtained from $\mathcal{H}_{m}$ as given in the Sec. 2.1.4 by the substitutions (51), (57) and (58). Thus, the substitution for $p_{3}$ is discontinuous near $\Sigma$. It could, therefore, seem that the corresponding Hamiltonian density $\bar{H}_{m}$ would not be continuous at $\Sigma$, but this is not true. The reason is that $\left.H_{m}\right|_{\Sigma}$ does not depend on $p_{3}$, if the conditions (49) are satisfied:

$$
\left.\frac{\partial H_{m}}{\partial p_{a}}\right|_{\Sigma}=\left.\dot{z}^{3}\right|_{\Sigma}=0
$$

Hence, we have from Eq. (24):

$$
\overline{\mathcal{H}}_{m}=-\int_{S} d S T_{0}^{0} .
$$

Collecting all results, we obtain finally:

$$
\check{\mathcal{H}}=\int_{S} d S\left(-T_{0}^{0}+\frac{1}{8 \pi \mathrm{G}} G_{0}^{0}\right)+\frac{1}{8 \pi \mathrm{G}} \int_{\partial S} d \partial S \sqrt{|\gamma|} L_{0}^{0} .
$$

This is the full 'off-shell' Hamiltonian of our system. If Einstein's equations hold, its value is just the surface integral.

Let us rewrite the volume integral in a covariant form. Any tensor density $W_{\nu}^{\mu}$ satisfies the identity

$$
W_{0}^{0}=\frac{1}{g^{00}}\left(W^{00}-g^{0 k} W_{k}^{0}\right) .
$$

The unit future-oriented normal covector $n_{\mu}$ to $S$ has the components $n_{\mu}=-N \delta_{\mu}^{0}$ with respect to the adapted coordinates; it follows that

$$
W_{0}^{0}=-\sqrt{q}\left(N w^{\perp \perp}+N^{k} w_{k}^{\perp}\right),
$$

where

$$
\begin{aligned}
w^{\perp \perp} & :=\frac{1}{\sqrt{|g|}} W^{\mu \nu} n_{\mu} n_{\nu}, \\
w_{k}^{\perp} & :=\frac{1}{\sqrt{|g|}} W_{k}^{\mu} n_{\mu} .
\end{aligned}
$$

Thus, the volume integral can be written as

$$
\int_{S} d S\left(-T_{0}^{0}+\frac{1}{8 \pi \mathrm{G}} G_{0}^{0}\right)=\frac{1}{8 \pi \mathrm{G}} \int_{S} d^{3} y \sqrt{q}\left(N C+N^{k} C_{k}\right),
$$

where

$$
C:=-\frac{1}{\sqrt{|g|}}\left(G^{\mu \nu}-8 \pi \mathrm{G} T^{\mu \nu}\right) n_{\mu} n_{\nu}
$$


and

$$
C_{k}:=-\frac{1}{\sqrt{|g|}}\left(G_{k}^{\mu}-8 \pi \mathrm{G} T_{k}^{\mu}\right) n_{\mu}
$$

are the super-Hamiltonian and the supermomentum of our system (or scalar and vector constraint functions).

Let us return to the formula (67), which not only implies the field equations, if we perform the variation on the L.H.S. and compare the result with the R.H.S., but it also determines the so-called control mode (see, eg. [13]) and the type of boundary value problem for the field equations. We observe that this mode is a kind of "curvature-control-mode"; it amounts to keeping fixed (controling) the external curvature $Q^{\alpha \beta}$ at the boundary $\Sigma^{+}$(see [14]). Such a boundary problem for Einstein's equations has not been studied. To pass to a more natural and in fact conventional approach, we have to perform an additional Legendre transformation at the boundary [14]:

$$
\mathcal{H}=\check{\mathcal{H}}-\frac{1}{16 \pi \mathrm{G}} \int_{\partial S} d^{2} \eta \gamma_{A B} Q^{A B}
$$

so that we have, using also Eq. (71), finally

$$
\mathcal{H}=\frac{1}{8 \pi \mathrm{G}} \int_{S} d S \sqrt{q}\left(N C+N^{k} C_{k}\right)-\frac{1}{16 \pi \mathrm{G}} \int_{\partial S} d \partial S Q^{00} \gamma_{00}
$$

and

$$
\begin{aligned}
\delta \mathcal{H} & =\int_{S^{-}} d S\left(\dot{z}^{a} \delta \bar{p}_{a}-\dot{\bar{p}}_{a} \delta z^{a}\right)+\frac{1}{16 \pi \mathrm{G}} \int_{S} d S\left(\dot{q}_{k l} \delta \pi^{k l}-\dot{\pi}^{k l} \delta q_{k l}\right) \\
& +\frac{1}{8 \pi \mathrm{G}} \int_{\partial S} d \partial S(\dot{\alpha} \delta \lambda-\dot{\lambda} \delta \alpha) \\
& +\frac{1}{16 \pi \mathrm{G}} \int_{\partial S} d \partial S\left(\gamma_{00} \delta Q^{00}+2 \gamma_{0 A} \delta Q^{0 A}-Q^{A B} \delta \gamma_{A B}\right) .
\end{aligned}
$$

The last surface integral in Eq. (74) will result in the A.D.M. energy, if the limit $\Sigma^{+} \rightarrow \infty$ is carefully performed (this has been shown in [14]). The last one in Eq. (75) defines the way of controle: $Q^{00}, Q^{0 A}$ and $\gamma_{A B}$ are kept fixed at the boundary.

The transformation to the Euler picture in the matter part of the Hamiltonian is straightforward; let us denote the resulting Hamiltonian density by $\bar{H}_{m}^{\prime}$. Most formulas of Sec. 2.1.5 will result in the analogous formulas for $\bar{H}_{m}^{\prime}$, if the substitutions (51), (57) and (58) are performed in them. For example, we have to use the modified formula (25), which will read at $\Sigma$ :

$$
T_{A}^{0}=-\bar{p}_{B} z_{A}^{B}, \quad T_{3}^{0}=-\bar{p}_{B} z_{3}^{B}-p(\bar{p}, z) z_{3}^{3},
$$

etc. Only those formulas that contain derivatives of $H_{m}^{\prime}$ with respect to the variables which are not contained in $\bar{H}_{m}^{\prime}$ (like $\left.p_{3}\right|_{\Sigma}$ and $\left.x_{A}^{3}\right|_{\Sigma}$ ) need some care to be properly transformed. 
We observe finally that the field equations derived from Eq. (75) (or an analogous equation of the Euler picture) will have the form of canonical equations. This is interesting, because Eq. (45) does not seem to have such form. The variations in Eq. (75) (or that of the Euler picture) must satisfy the boundary conditions (49) (or (50)) and will, therefore, lead to trivial surface equations; the volume equations alone have the canonival form. For example, the would be counterpart of Eq. (45) originates from the term

$$
\left.\frac{\partial \bar{H}_{m}^{\prime}}{\partial x_{3}^{k}} \delta x^{k}\right|_{\Sigma}
$$

in the variation of $\bar{H}_{m}^{\prime}$. As $\left.\delta x^{3}\right|_{\Sigma}=0$, the only equation which is implied thereby reads

$$
\left.\frac{\partial \bar{H}_{m}^{\prime}}{\partial x_{3}^{A}}\right|_{\Sigma}=0 .
$$

Further, a counterpart of Eq. (46) is valid for this derivative, so the above equation is equivalent to

$$
\left.z_{\mu}^{3} T_{A}^{\mu}\right|_{\Sigma}=0 .
$$

Moreover, $\left.z_{B}^{3}\right|_{\Sigma}=0$, so the equation reduces to $\left.T_{A}^{3}\right|_{\Sigma}=0$. However, these components of $T_{\nu}^{\mu}$ vanish identically at $\Sigma$, besause $\left.u^{3}\right|_{\Sigma}=0$ (cf. Eq. (6)). Thus, there is no surface field equation.

\section{Fluid shell}

In this section, we are going to describe the dynamics of a delta-function distribution of fluid. The matter will be coupled to the dynamical gravity from the start. We shall consider a special case: just one shell in vacuum; a generalization to more shells surrounded by a piecewise smooth matter is straightforward as far as the shells do not intersect.

\subsection{Action in the volume form}

The shell can be represented as a delta-function singularity in the mole density $h$. The action can then be written as a volume integral of the same form as for a regular distribution of matter. This holds also for the gravitational part. We shall give a more detailed description of this volume form and then transform it to a combinations of volume and surface integrals, where no delta-functions will feature. This may be useful, because much more general choice of coordinates is then allowed. Indeed, the $\delta$-function method works only if the coordinates are such that the corresponding components of the four-metric are continuous. Further, the 
coordinate position of the shell - the three-surface in the spacetime $M$ and the twosurface in the matter space $Z$ - will be kept fixed. Here, everything can be repeated what has already been said in Sec. 2.2.1 about this point.

The matter space $Z$ remains, therefore, three-dimensional first. Let $\Sigma_{z}$ be a twodimensional surface in $Z$ on which the matter is concentrated. Let $z^{a}$ be coordinates adapted to $\Sigma_{z}$ so that the equation $z^{3}=z_{0}^{3}$ determines $\Sigma_{z}$. Such coordinates are determined up to a transformation

$$
\begin{array}{ll}
z^{1}=z^{\prime 1}\left(z^{a}\right), & z^{\prime 2}=z^{\prime 2}\left(z^{a}\right), \\
z^{\prime 3}=z^{\prime 3}\left(z^{a}\right), & z_{0}^{\prime 3}=z^{\prime 3}\left(z^{A}, z_{0}^{3}\right),
\end{array}
$$

where $z_{0}^{\prime 3}$ is a constant independent of $z^{A}$. Then,

$$
\frac{\partial\left(z^{1}, z^{2}, z^{3}\right)}{\partial\left(z^{1}, z^{\prime 2}, z^{\prime 3}\right)}=\frac{\partial\left(z^{1}, z^{2}\right)}{\partial\left(z^{\prime 1}, z^{\prime 2}\right)} \cdot \frac{\partial z^{3}}{\partial z^{\prime 3}} .
$$

We decompose the molar density $h$ in the adapted coordinates as follows

$$
h=h_{s} \delta\left(z^{3}-z_{0}^{3}\right)
$$

where $h_{s}\left(z^{1}, z^{2}\right)$ is a two-dimesional density on $\Sigma_{z}$. If we change the adapted coordinates, we have

$$
\tilde{h}=\frac{\partial\left(z^{1}, z^{2}, z^{3}\right)}{\partial\left(\tilde{z}^{1}, \tilde{z}^{2}, \tilde{z}^{3}\right)} h=h_{s} \frac{\partial\left(z^{1}, z^{2}\right)}{\partial\left(\tilde{z}^{1}, \tilde{z}^{2}\right)} \cdot \delta\left(z^{3}-z_{0}^{3}\right) \frac{\partial z^{3}}{\partial \tilde{z}^{3}}=\tilde{h}_{s} \cdot \delta\left(\tilde{z}^{3}-\tilde{z}_{0}^{3}\right),
$$

so the decomposition is independent of the choice of adapted coordinates, and defines, in fact, a two-dimensional matter space $\Sigma_{z}$ with a two-dimesional mole density $h_{s}$; later, we will pass to this space.

In the spacetime $M$ with coordinates $x^{\mu}$, the matter fields are $z^{a}\left(x^{\mu}\right)$; the shell occupies a three-dimensional surface $\Sigma$, which can be described by the embedding functions $x^{\mu}=x^{\mu}\left(\xi^{\alpha}\right), \alpha=0,1,2$, or by means of the equation $z^{3}\left(x^{\mu}\right)=z_{0}^{3}$. Later, we will pass to the the matter fields $z^{A}=z^{A}\left(\xi^{\alpha}\right)$, where $z^{A}\left(\xi^{\alpha}\right)=z^{A}\left(x^{\mu}\left(\xi^{\alpha}\right)\right)$.

The gravitational field is described by the metric $g_{\mu \nu}(x)$; we require:

Condition 1' the spacetime $(M, g)$ is asymptotically flat and globally hyperbolic;

Condition 2' there are coordinates $x^{\mu}$ in a neighbourhood of each point of $\Sigma$ such that the metric $g_{\mu \nu}(x)$ is $C^{0}$ everywhere, piecewise $C^{\infty}$, so that the only discontinuity is a jump in the first derivatives at $\Sigma$.

The second derivatives of the metric will then have a delta-function singularity at $\Sigma$ so that the Einstein's equations can be satisfied. 
The total action for the system consisting of the shell and the gravitational field can then be written in the following form

$$
I=\frac{1}{16 \pi \mathrm{G}} \int_{M} d^{4} x \sqrt{|g|} R-\int_{M} d^{4} x \sqrt{|g|} n e(n) .
$$

One can use this volume form of the action to derive the equations of motion. However, there is also a 'surface form' of the action; we are going to derive this one in the next section.

\subsection{The surface form of the action}

The coordinates satisfying Condition 2' are not uniquely determined. We can use this freedom for the derivation of the surface form; the tool will be the adapted spacetime coordinates $x^{\mu}$, defined by the property

$$
x^{3}=z^{3}(x), \quad \xi^{\alpha}=\left.x^{\alpha}\right|_{\Sigma}
$$

Then, the induced metric $\gamma_{\alpha \beta}$ on the shell is

$$
\gamma_{\alpha \beta}=\left.g_{\alpha \beta}\right|_{\Sigma}
$$

and its determinant $\gamma$ is related to the determinant $g$ of the four-metric $g_{\mu \nu}$ by

$$
\gamma=g \cdot g^{33}
$$

\subsubsection{Matter action}

Formula (11) together with Eq. (76) give

$$
j^{\mu}=h_{s} \delta\left(z^{3}-z_{0}^{3}\right) \epsilon^{\mu \nu \rho \sigma} z_{\nu}^{1} z_{\rho}^{2} z_{\sigma}^{3}
$$

Hence, $j^{3}=0$, and as $\delta\left(z^{3}-z_{0}^{3}\right)=z_{3}^{3} \delta\left(x^{3}-x_{0}^{3}\right), j^{\alpha}$ can be written as

$$
j^{\alpha}=j_{s}^{\alpha} \delta\left(x^{3}-x_{0}^{3}\right)
$$

The mole density $n$ can be calculated from Eq. (3). We obtain

$$
\sqrt{-g_{\mu \nu} j^{\mu} j^{\nu}}=\sqrt{-g_{\alpha \beta} j_{s}^{\alpha} j_{s}^{\beta}} \delta\left(x^{3}-x_{0}^{3}\right)=\sqrt{-\gamma_{\alpha \beta} j_{s}^{\alpha} j_{s}^{\beta}} \delta\left(x^{3}-x_{0}^{3}\right),
$$

and Eq. (79) yields:

$$
n=\frac{1}{\sqrt{|\gamma|}} \sqrt{-\gamma_{\alpha \beta} j_{s}^{\alpha} j_{s}^{\beta}} \sqrt{g^{33}} \delta\left(x^{3}-x_{0}^{3}\right)
$$


We define the surface mole density $n_{s}$ by

$$
n_{s}=\frac{1}{\sqrt{|\gamma|}} \sqrt{-\gamma_{\alpha \beta} j_{s}^{\alpha} j_{s}^{\beta}}
$$

so that

$$
n=n_{s} \sqrt{g^{33}} \delta\left(x^{3}-x_{0}^{3}\right),
$$

where $\sqrt{g^{33}} \delta\left(x^{3}-x_{0}^{3}\right)$ is already a scalar with respect the reparametrizations of $x^{3}$. For the velocity $u^{\mu}$, we have the expansion

$$
u^{\mu}=v^{\alpha} e_{\alpha}^{\mu}
$$

where

$$
e_{\alpha}^{\mu}:=\frac{\partial x^{\mu}}{\partial \xi^{\alpha}}
$$

so that

$$
\gamma_{\alpha \beta} v^{\alpha} v^{\beta}=-1
$$

and

$$
j_{s}^{\alpha}=\sqrt{|\gamma|} n_{s} v^{\alpha}, \quad j_{s}^{\alpha}=h_{s} \epsilon^{\alpha \beta \gamma} z_{\beta}^{1} z_{\gamma}^{2}
$$

Then the transcription of the matter Lagrangian density is straightforward:

$$
L_{m}=-\sqrt{-g} n e(n)=-\sqrt{|\gamma|} n_{s} e_{s}\left(n_{s}\right) \delta\left(x^{3}-x_{0}^{3}\right),
$$

where $e_{s}\left(n_{s}\right)$ is the energy per mole of the shell matter. We define the surface Lagrange density $L_{s}$ and the surface mass density $\rho_{s}$ by

$$
L_{m}:=L_{s} \delta\left(x^{3}-x_{0}^{3}\right), \quad \rho_{s}:=n_{s} e_{s}\left(n_{s}\right),
$$

so that

$$
L_{s}=-\sqrt{|\gamma|} \rho_{s}\left(n_{s}\right)
$$

If we perform the trivial integration over $x^{3}$ in $I_{m}$, the matter action becomes a surface integral

$$
I_{m}=-\int_{\Sigma} d^{3} \xi \sqrt{|\gamma|} n_{s} e_{s}\left(n_{s}\right)
$$

This expression is invariant under the transformation of coordinates at the shell.

The action $I_{m}$ can be varied with respect to the shell metric $\gamma_{\alpha \beta}$ with the result

$$
\delta I_{m}=\int_{\Sigma} d^{3} \xi\left(-\frac{1}{2} \sqrt{|\gamma|} \gamma^{\alpha \beta} \rho_{s}-\sqrt{|\gamma|} \rho_{s}^{\prime} \frac{\partial n_{s}}{\partial \gamma_{\alpha \beta}}\right) \delta \gamma_{\alpha \beta} .
$$

In analogy with the formula (5), we obtain

$$
\frac{\partial n_{s}}{\partial \gamma_{\alpha \beta}}=-\frac{1}{2} n_{s}\left(\gamma_{\alpha \beta}+v^{\alpha} v^{\beta}\right)
$$


and we have

$$
\delta I_{m}=\frac{1}{2} \int_{\Sigma} d^{3} \xi T_{s}^{\alpha \beta} \delta \gamma_{\alpha \beta}
$$

where $T_{s}^{\alpha \beta}$ is the surface stress-energy tensor,

$$
T_{s}^{\alpha \beta}:=\sqrt{|\gamma|}\left(\rho_{s} v^{\alpha} v^{\beta}-\sigma\left(\gamma_{\alpha \beta}+v^{\alpha} v^{\beta}\right)\right)
$$

and

$$
\sigma:=n_{s} \rho_{s}^{\prime}-\rho_{s}=-n_{s}^{2} e_{s}^{\prime}
$$

is the surface tension (negative two-dimensional presure). Moreover, it holds that

$$
T^{\mu \nu}=\frac{\partial x^{\mu}}{\partial \xi^{\alpha}} \frac{\partial x^{\nu}}{\partial \xi^{\beta}} T_{s}^{\alpha \beta} \sqrt{g^{33}} \delta\left(x^{3}-x_{0}^{3}\right) .
$$

We obtain easily relations analogous to the Eqs. (11), (12) and (13):

$$
T_{s}^{\alpha \beta}(\xi)=2 \frac{\partial L_{s}}{\partial \gamma_{\alpha \beta}(\xi)}
$$

(this is a form of Eq. (88));

$$
\frac{\partial L_{s}}{\partial z_{\alpha}^{A}} z_{\beta}^{A}+2 \frac{\partial L_{s}}{\partial \gamma_{\alpha \gamma}} \gamma_{\beta \gamma}=L_{s} \delta_{\beta}^{\alpha},
$$

because $L_{s}$ is a three-density on $\Sigma$, and the Noether identity

$$
T_{s \beta}^{\alpha}=L_{s} \delta_{\beta}^{\alpha}-\frac{\partial L_{s}}{\partial z_{\alpha}^{A}} z_{\beta}^{A} .
$$

\subsubsection{Gravitation action}

The next task is to rewrite the shell part of the gravitational action $I_{g}$ in the surface form. The following lemma is vital.

Lemma 3 In the adapted coordinates $x^{\mu}$ that satisfy Condition 2', the delta-function part of the gravitational Lagrange density is given by

$$
\frac{1}{16 \pi G} \sqrt{|g|} R=-\frac{1}{8 \pi G} \sqrt{|\gamma|}[L] \delta\left(x^{3}-x_{0}^{3}\right)+\ldots
$$

where the dots represent regular terms, $L=\gamma_{\alpha \beta} L^{\alpha \beta}, L^{\alpha \beta}$ is the second fundamental form of $\Sigma$ corresponding to the normal oriented outwards of $V^{-}$and the abbreviation $[f]:=f_{+}-f_{-}$for the jump of a quantity $f$ accross the shell is used. 
The proof of the lemma is relegated to the Appendix. Eq. (92) implies immediately that the gravitational action can be transformed to

$$
I_{g}=\frac{1}{16 \pi \mathrm{G}} \int_{V^{+} \cup V^{-}} d^{4} x \sqrt{|g|} R-\frac{1}{8 \pi \mathrm{G}} \int_{\Sigma} d^{3} \xi \sqrt{|\gamma|}[L] .
$$

Eqs. (85) and (93) give the total action in the surface form:

$$
\begin{aligned}
I & =\frac{1}{16 \pi \mathrm{G}} \int_{V^{-}} d^{4} x \sqrt{|g|} R+\frac{1}{16 \pi \mathrm{G}} \int_{V^{+}} d^{4} x \sqrt{|g|} R \\
& -\frac{1}{8 \pi \mathrm{G}} \int_{\Sigma} d^{3} \xi \sqrt{|\gamma|}[L]-\int_{\Sigma} d^{3} \xi \sqrt{|\gamma|} n_{s} e_{s}\left(n_{s}\right) .
\end{aligned}
$$

This action functional is equivalent to that given by Eq. (77), if the coordinates satisfy Condition 2'. It has, however, two advantages in comparison with (77):

1. all integrands in (94) are smooth,

2. it is valid and can be used with more general coordinates, namely arbitrary smooth coordinates $x_{ \pm}^{\mu}$ within $V^{ \pm}$and arbitrary coordinates $\xi^{\alpha}$ within $\Sigma$.

The fields in the action (94) are the matter fields $z^{A}(y)$ on $\Sigma$ (observe that the fictitious field $z^{3}$ disappeared from the action), the gravity fields $g_{\mu \nu}(x)$ in $V^{ \pm}$and $\gamma_{\alpha \beta}(y)$ in $\Sigma$. The metric has to satisfy the so-called continuity relations

$$
\gamma_{\alpha \beta}(\xi)=\left(g_{\mu \nu}^{-} \frac{\partial x_{-}^{\mu}}{\partial \xi^{\alpha}} \frac{\partial x_{-}^{\nu}}{\partial \xi^{\beta}}\left(x_{-}(\xi)\right)\right)^{-}=\left(g_{\mu \nu}^{+} \frac{\partial x_{+}^{\mu}}{\partial \xi^{\alpha}} \frac{\partial x_{+}^{\nu}}{\partial \xi^{\beta}}\left(x_{+}(\xi)\right)\right)^{+}
$$

where the symbols ()$^{ \pm}$denote the limits from the volumes $V^{ \pm}$towards $\Sigma$. The role of the continuity relations (95) is to define the configuration space of our system similarly as a control mode or some fall-off conditions do. The embedding functions $x_{ \pm}^{\mu}(\xi)$ are fixed; their variation is zero.

We also have to specify the integration volumes; this will be done in analogy to Sec. 2.2.1: the volume $V$ is chosen to be bounded by two Cauchy surfaces, $S_{1}$ and $S_{2}$, and by a timelike surface $\Sigma^{+}$(which will be eventually pushed to the infinity); the surface $\Sigma$ separates $V$ in two parts, $V^{ \pm}$, and the surfaces $S_{i}$ into $S_{i}^{ \pm}$; the intersections of $\Sigma$ with $S_{i}$ will be denoted by $\partial \Sigma_{i}$ and we will assume that they together form the complete boundary of $\Sigma$; the intersections of $\Sigma^{+}$with $S_{i}$ will be denoted by $\partial S_{i}$ and we will assume that they form the complete boundary of $\Sigma^{+}$.

This form of the action will be our starting point to the derivation of the field equations as well as the Hamiltonian formalism. 


\subsection{The variational formula}

The variation of the matter part $I_{m}$ of the action (94), if we calculate in the coordinates $\xi^{\alpha}$ that are adapted to the surfaces $\partial \Sigma_{i}$ by $\xi^{0}=t_{i}$ and $\eta^{A}=\xi^{A}$ at $\partial \Sigma_{i}$, is

$$
\begin{aligned}
\delta I_{m} & =\int_{\Sigma} d^{3} \xi\left(\frac{\partial L_{s}}{\partial z^{A}}-\partial_{\alpha} \frac{\partial L_{s}}{\partial z_{\alpha}^{A}}\right) \delta z^{A}+\frac{1}{2} \int_{\Sigma} d^{3} \xi T_{s}^{\alpha \beta} \delta \gamma_{\alpha \beta} \\
& +\int_{\partial \Sigma_{2}} d^{2} \eta \frac{\partial L_{s}}{\partial z_{0}^{A}} \delta z^{A}-\int_{\partial \Sigma_{1}} d^{2} \eta \frac{\partial L_{s}}{\partial z_{0}^{A}} \delta z^{A}
\end{aligned}
$$

We define the matter momenta $p_{A}$ by

$$
p_{A}:=\frac{\partial L_{s}}{\partial z_{0}^{A}}
$$

As $p_{A}$ is a well-defined two-surface density (cf. the discussion below Eq. (21)), we obtain the covariant formula:

$$
\begin{aligned}
\delta I_{m} & =\int_{\Sigma} d^{3} \xi\left(\frac{\partial L_{s}}{\partial z^{A}}-\partial_{\alpha} \frac{\partial L_{s}}{\partial z_{\alpha}^{A}}\right) \delta z^{A}+\frac{1}{2} \int_{\Sigma} d^{3} \xi T_{s}^{\alpha \beta} \delta \gamma_{\alpha \beta} \\
& +\int_{\partial \Sigma_{2}} d^{2} \eta p_{A} \delta z^{A}-\int_{\partial \Sigma_{1}} d^{2} \eta p_{A} \delta z^{A} .
\end{aligned}
$$

To calculate the variation of the gravitational part $I_{g}$ of the action (94), we first rewrite the surface integral in $I_{g}$ with the help of the trace part of the Eq. (54),

$$
Q=2 \sqrt{|\gamma|} L
$$

as

$$
-\frac{1}{8 \pi \mathrm{G}} \int_{\Sigma} d^{3} \xi \sqrt{|\gamma|}[L]=-\frac{1}{16 \pi \mathrm{G}} \int_{\Sigma} d^{3} \xi \gamma_{\alpha \beta}\left[Q^{\alpha \beta}\right]
$$

so that

$$
\delta\left(-\frac{1}{8 \pi \mathrm{G}} \int_{\Sigma} d^{3} \xi \sqrt{|\gamma|}[L]\right)=-\frac{1}{16 \pi \mathrm{G}} \int_{\Sigma} d^{3} \xi\left(\delta \gamma_{\alpha \beta}\left[Q^{\alpha \beta}\right]+\gamma_{\alpha \beta} \delta\left[Q^{\alpha \beta}\right]\right) .
$$

Then, we apply Lemma 1 (Eqs. (52) and (54)) and Eq. (98) with the result:

$$
\begin{aligned}
\delta I_{g} & =-\frac{1}{16 \pi \mathrm{G}} \int_{V^{-}} d^{4} x G^{\mu \nu} \delta g_{\mu \nu}-\frac{1}{16 \pi \mathrm{G}} \int_{V^{+}} d^{4} x G^{\mu \nu} \delta g_{\mu \nu} \\
& -\frac{1}{16 \pi \mathrm{G}} \int_{\Sigma} d^{3} \xi\left[Q^{\alpha \beta}\right] \delta \gamma_{\alpha \beta} \\
& -\frac{1}{16 \pi \mathrm{G}} \int_{S_{2}} d^{3} y q_{k l} \delta \pi^{k l}+\frac{1}{16 \pi \mathrm{G}} \int_{S_{1}} d^{3} y q_{k l} \delta \pi^{k l} \\
& -\frac{1}{8 \pi \mathrm{G}} \int_{\partial \Sigma_{2}} d^{2} \eta \lambda \delta[\alpha]+\frac{1}{8 \pi \mathrm{G}} \int_{\partial \Sigma_{1}} d^{2} \eta \lambda \delta[\alpha] \\
& +\frac{1}{8 \pi \mathrm{G}} \int_{\partial S_{2}} d^{2} \eta \lambda \delta \alpha-\frac{1}{8 \pi \mathrm{G}} \int_{\partial S_{1}} d^{2} \eta \lambda \delta \alpha-\frac{1}{16 \pi \mathrm{G}} \int_{\Sigma} d^{3} \xi \gamma_{\alpha \beta} \delta Q^{\alpha \beta}
\end{aligned}
$$


Here, $q_{k l}(y)$ and $\delta q_{k l}(y)$ are continuous along $S_{i}$, but $\pi^{k l}(y)$ and $\delta \pi^{k l}(y)$ have a jump at $\partial \Sigma_{i}$. Eqs. (96) and (99) imply the following formula for the total action:

$$
\begin{aligned}
\delta I & =-\frac{1}{16 \pi \mathrm{G}} \int_{V^{-}} d^{4} x G^{\mu \nu} \delta g_{\mu \nu}-\frac{1}{16 \pi \mathrm{G}} \int_{V^{+}} d^{4} x G^{\mu \nu} \delta g_{\mu \nu} \\
& +\int_{\Sigma} d^{3} \xi\left(\frac{\partial L_{s}}{\partial z^{A}}-\partial_{\alpha} \frac{\partial L_{s}}{\partial z_{\alpha}^{A}}\right) \delta z^{A}+\frac{1}{16 \pi \mathrm{G}} \int_{\Sigma} d^{3} \xi\left(8 \pi \mathrm{G} T_{s}^{\alpha \beta}-\left[Q^{\alpha \beta}\right]\right) \delta \gamma_{\alpha \beta} \\
& -\frac{1}{16 \pi \mathrm{G}} \int_{S_{2}} d^{3} y q_{k l} \delta \pi^{k l}+\frac{1}{16 \pi \mathrm{G}} \int_{S_{1}} d^{3} y q_{k l} \delta \pi^{k l} \\
& -\frac{1}{8 \pi \mathrm{G}} \int_{\partial \Sigma_{2}} d^{2} \eta \sqrt{\lambda} \delta[\alpha]+\frac{1}{8 \pi \mathrm{G}} \int_{\partial \Sigma_{1}} d^{2} \eta \sqrt{\lambda} \delta[\alpha] \\
& +\int_{\partial \Sigma_{2}} d^{2} \eta p_{A} \delta z^{A}-\int_{\partial \Sigma_{1}} d^{2} \eta p_{A} \delta z^{A} \\
& +\frac{1}{8 \pi \mathrm{G}} \int_{\partial S_{2}} d^{2} \eta \sqrt{\lambda} \delta \alpha-\frac{1}{8 \pi \mathrm{G}} \int_{\partial S_{1}} d^{2} \eta \sqrt{\lambda} \delta \alpha-\frac{1}{16 \pi \mathrm{G}} \int_{\Sigma} d^{3} \xi \gamma_{\alpha \beta} \delta Q^{\alpha \beta} .(100)
\end{aligned}
$$

From the variational formula (100), we can read off the field equations: in $V^{-}$ and $V^{+}$,

$$
G^{\mu \nu}=0
$$

at $\Sigma$, we obtain

$$
\begin{aligned}
\frac{\partial L_{s}}{\partial z^{A}}-\partial_{\alpha} \frac{\partial L_{s}}{\partial z_{\alpha}^{A}} & =0 \\
{\left[Q^{\alpha \beta}\right] } & =8 \pi \mathrm{G} T_{s}^{\alpha \beta} .
\end{aligned}
$$

Eq. (103) is the well-known dynamical equation for thin shells [11]; we shall refer to it as 'Israel's equation'. It may be considered as the singular part of Einstein equations, corresponding to $\delta$-like sources. Eq. (102) can be interpreted as a threedimensional stress-energy conservation: we can show in an analogous way as in Sec. 2.1 .2 that

$$
\nabla_{\alpha} T_{s \beta}^{\alpha}=\left(\frac{\partial L_{s}}{\partial z^{A}}-\partial_{\alpha} \frac{\partial L_{s}}{\partial z_{\alpha}^{A}}\right) z_{\beta}^{A},
$$

where $\nabla_{\alpha}$ is a covariant derivative associated with the metric $\gamma_{\alpha \beta}$. This identity implies that Eq. (102) is equivalent to

$$
\nabla_{\alpha} T_{s \beta}^{\alpha}=0
$$

(which comprises only two independent equations).

Eq. (100) implies a generating formula for the field equations analogous to Eq. (22). In order to derive this formula, we first have to introduce a foliation of the integration volume in (100). This is an arbitrary smooth family of spacelike surfaces $S_{t}$ such that $S_{i}=S_{t_{i}}$; we allow for the surfaces $S_{t}$ having a cusp at $\partial \Sigma_{t}=\Sigma \cap S_{t}$ so 
that the normal $n^{\mu}$ can have a step discontinuity there. This leads to jumps in $\pi^{k l}$ and $\alpha$ across $\partial \Sigma_{t}$.

We also have to introduce adapted cordinates $x^{\mu}$ so that the surfaces $S_{t}$ are given by $x^{0}=t, \Sigma$ by $x^{3}=0$ and $\Sigma^{+}$by $x^{3}=r^{+}$; further, $y^{k}=\left.x^{k}\right|_{S_{t}}, \xi^{\alpha}=\left.x^{\alpha}\right|_{\Sigma}$, $\xi^{\alpha}=\left.x^{\alpha}\right|_{\Sigma^{+}}, \eta^{A}=\left.x^{A}\right|_{\partial \Sigma_{t}}$ and $\eta^{A}=\left.x^{A}\right|_{\partial S_{t}}$. Observe that the full four-metric need not be continuous across $\Sigma$ with respect to these coordinates. Observe that $t=$ const is a continuous surface intersecting $\Sigma$ and $\Sigma^{+}$, and $\partial / \partial t$ is a continuous vector field everywhere.

Eq. (94) and

$$
I=\int_{t_{1}}^{t_{2}} d t \mathcal{L}
$$

imply for $\mathcal{L}$ :

$$
\begin{aligned}
\mathcal{L} & =\frac{1}{16 \pi \mathrm{G}} \int_{S_{t}^{-}} d^{3} y \sqrt{|g|} R+\frac{1}{16 \pi \mathrm{G}} \int_{S_{t}^{+}} d^{3} y \sqrt{|g|} R \\
& -\frac{1}{8 \pi \mathrm{G}} \int_{\partial S_{t}^{-}} d^{2} \eta \sqrt{|\gamma|}[L]-\int_{\partial S_{t}^{-}} d^{2} \eta \sqrt{|\gamma|} n_{s} e_{s}\left(n_{s}\right) .
\end{aligned}
$$

If we rewrite Eq. (100) in the form

$$
\delta I=\int_{t_{1}}^{t_{2}} d t \delta \mathcal{L}
$$

we obtain the variation formula for our system:

$$
\begin{aligned}
\delta \mathcal{L} & =-\frac{1}{16 \pi \mathrm{G}} \int_{S_{t}} d^{3} y\left(q_{k l} \delta \pi^{k l}\right) \cdot-\frac{1}{8 \pi \mathrm{G}} \int_{\partial \Sigma_{t}} d^{2} \eta(\lambda \delta[\alpha]) \\
& +\int_{\partial \Sigma_{t}} d^{2} \eta\left(p_{A} \delta z^{A}\right)^{\cdot}+\frac{1}{8 \pi \mathrm{G}} \int_{\partial S_{t}} d^{2} \eta(\lambda \delta \alpha)^{\cdot} \\
& -\frac{1}{16 \pi \mathrm{G}} \int_{\partial S_{t}} d^{2} \eta \gamma_{\alpha \beta} \delta Q^{\alpha \beta} .
\end{aligned}
$$

Performing the variation in (104) and comparing the result with the R.H.S. of (105) recovers the definition of momenta and the field equations.

\subsection{The Legendre transformation}

Let us define the Hamiltonian in analogous way to Sec. 2.2.3:

$$
\begin{aligned}
\check{\mathcal{H}} & =-\mathcal{L}-\frac{1}{16 \pi \mathrm{G}} \int_{S} d^{3} y q_{k l} \dot{\pi}^{k l}-\frac{1}{8 \pi \mathrm{G}} \int_{S \cap \Sigma} d^{2} \eta \sqrt{\lambda}[\dot{\alpha}] \\
& +\frac{1}{8 \pi \mathrm{G}} \int_{S \cap \Sigma^{+}} d^{2} \eta \sqrt{\lambda} \dot{\alpha}+\int_{S \cap \Sigma} d^{2} \eta p_{A} \dot{z}^{A},
\end{aligned}
$$

where $[\alpha]:=-\alpha_{+}-\alpha_{-}$at $\Sigma, \alpha_{+}$is defined by the normal to $\Sigma$ that is outward to $V^{+}$and the future normal to $S^{-}, \alpha_{+}$is defined by the normal to $\Sigma$ that is outward to $V^{-}$and the future normal to $S^{+}$. 
To calculate the variation of $\check{\mathcal{H}}$, we have to regroup terms in Eq. (105)

$$
\begin{aligned}
\delta \mathcal{L} & =\frac{1}{16 \pi \mathrm{G}}\left[-\int_{S^{-}} d^{3} y\left(q_{k l} \delta \pi^{k l}\right)^{\cdot}+2 \int_{S^{-} \cap \Sigma} d^{2} \eta\left(\sqrt{\lambda} \delta \alpha_{-}\right)^{\cdot}\right] \\
& +\frac{1}{16 \pi \mathrm{G}}\left[-\int_{S^{+}} d^{3} y\left(q_{k l} \delta \pi^{k l}\right)^{\cdot}+2 \int_{S^{+} \cap \Sigma} d^{2} \eta\left(\sqrt{\lambda} \delta \alpha_{+}\right)^{\cdot}\right. \\
& \left.+2 \int_{S^{+} \cap \Sigma^{+}} d^{2} \eta(\sqrt{\lambda} \delta \alpha)^{\cdot}\right]+\int_{S \cap \Sigma} d^{2} \eta\left(p_{A} \delta z^{A}\right) \cdot-\frac{1}{16 \pi \mathrm{G}} \int_{\partial S} d^{2} \eta \gamma_{\alpha \beta} \delta Q^{\alpha \beta} .
\end{aligned}
$$

Then we vary Eq. (106), substitute for $\delta \mathcal{L}$ the regrouped expression, and apply Lemma 1 to each of the two volumes $V^{ \pm}$; the result is

$$
\begin{aligned}
\delta \check{\mathcal{H}} & =-\frac{1}{16 \pi \mathrm{G}} \int_{S} d^{3} y\left(\dot{\pi}^{k l} \delta q_{k l}-\dot{q}_{k l} \delta \pi^{k l}\right)+\frac{1}{16 \pi \mathrm{G}} \int_{S \cap \Sigma} d^{2} \eta \sqrt{\lambda}\left(\frac{\dot{\lambda}}{\lambda} \delta[\alpha]-[\dot{\alpha}] \frac{\delta \lambda}{\lambda}\right) \\
& -\int_{S \cap \Sigma} d^{2} \eta\left(\dot{p}_{A} \delta z^{A}-\dot{z}^{A} \delta p_{A}\right)-\frac{1}{16 \pi \mathrm{G}} \int_{S \cap \Sigma^{+}} d^{2} \eta \sqrt{\lambda}\left(\frac{\dot{\lambda}}{\lambda} \delta \alpha-\dot{\alpha} \frac{\delta \lambda}{\lambda}\right) \\
& +\frac{1}{16 \pi \mathrm{G}} \int_{S \cap \Sigma^{+}} d^{2} \eta \gamma_{\alpha \beta} \delta Q^{\alpha \beta} .
\end{aligned}
$$

This is the Hamiltonian variation formula for the field equations.

Let us compute the value of the Hamiltonian. To this aim, we insert from Eqs. (94) and (97) into Eq. (106) and apply Lemma 2. A simple calculation leads to

$$
\begin{aligned}
\check{\mathcal{H}} & =\frac{1}{8 \pi \mathrm{G}} \int_{S^{-}} d^{3} y G_{0}^{0}+\frac{1}{8 \pi \mathrm{G}} \int_{S^{+}} d^{3} y G_{0}^{0}+\frac{1}{8 \pi \mathrm{G}} \int_{S \cap \Sigma} d^{2} \eta\left[Q_{0}^{0}\right] \\
& -\int_{S \cap \Sigma} d^{2} \eta T_{s 0}^{0}+\frac{1}{8 \pi \mathrm{G}} \int_{S \cap \Sigma^{+}} d^{2} \eta L_{0}^{0} .
\end{aligned}
$$

The term $-\frac{1}{8 \pi \mathrm{G}}\left[Q_{0}^{0}\right]$ has an interesting interpretation. It has been obtained as the sum

$$
-\frac{1}{8 \pi \mathrm{G}}\left[Q_{0}^{0}\right]=-\frac{1}{16 \pi \mathrm{G}}[Q]+\frac{1}{8 \pi \mathrm{G}}\left[L_{0}^{0}\right] .
$$

The first summand is the delta-function term in the gravitational Lagrangian density in the volume form,

$$
\frac{1}{16 \pi \mathrm{G}} \sqrt{|g|} R
$$

and the meaning of the second is given by the following lemma:

Lemma 4 If the four-metric is continuous in the adapted coordinates, then we have at $\Sigma$ :

$$
-\frac{1}{8 \pi G} \sqrt{|g|} R_{0}^{0}=-\frac{1}{8 \pi G}\left[L_{0}^{0}\right] \delta\left(x^{3}-x_{0}^{3}\right)+\ldots,
$$

where the dots represent regular terms.

Thus, the term is the delta-function part of the expression

$$
-\frac{1}{8 \pi \mathrm{G}} G_{0}^{0}
$$


(cf. (53)). If the assumptions of Lemma 4 are satisfied, then the three first integrals on the R.H.S. of (107) can be written in volume form just as

$$
-\frac{1}{8 \pi \mathrm{G}} \int_{S} d^{3} y G_{0}^{0}
$$

The Legendre transformation at $\Sigma^{+}$similar to that in Sec. 2.2.3 can be performed exactly as in Sec. 2.2.3. The transformation to the Euler picture in the matter part of the shell Hamiltonian (106) is much simpler than the analogous transformation of the step Hamiltonian (66), because all formulas of Sec. 2.1.5 remain valid, they must only be rewritten in three spacetime and two matter space dimensions.

\subsection{The form of the Hamiltonian}

In this section, the Hamiltonian (108) will be expressed as a functional of the canonical variables $N, N_{k}, q_{k l}, \pi^{k l}, \lambda$ and $\alpha$.

Observe that the formulas (107) and (108) are valid in any coordinates that are adapted to the foliation and that make the embedding formulas for $\Sigma$ and $\Sigma^{+}$time independent. More specifically, the coordinate $t$ must be constant along the surfaces $S$, the embedding formulas for $\Sigma$ and $\Sigma^{+}$must read

$$
t=\xi^{0}, \quad y_{ \pm}^{k}=y_{ \pm}^{k}\left(\xi^{K}\right)
$$

and the embedding formulas for $S \cap \Sigma$ in $\Sigma$ is

$$
\xi^{0}=\text { const }, \quad \xi^{K}=\eta^{K}
$$

Let us recall that one important point of our method is that the boundaries are time independent in the above sense and their variations are zero.

The $2+1$ decomposition of the metric $\gamma_{\alpha \beta}$ at $\Sigma$ and $\Sigma^{+}$is analogous to that of $g_{\mu \nu}$. In particular, we define the (surface) lapse $\nu$ and the (surface) shift $\nu_{K}$ by

$$
\gamma^{\alpha \beta}=\left(\begin{array}{ll}
-\frac{1}{\nu^{2}}, & \frac{\nu^{L}}{\nu^{2}} \\
\frac{\nu^{K}}{\nu^{2}}, & \lambda^{K L}-\frac{\nu^{K} \nu^{L}}{\nu^{2}}
\end{array}\right)
$$

so that

$$
\gamma_{00}=-\nu^{2}+\lambda^{K L} \nu_{K} \nu_{L}
$$

the $2+1$ deconposition of the continuity relations (95) reads

$$
\begin{aligned}
\nu & =\sqrt{N_{ \pm}^{2}-\left(N_{ \pm}^{\perp}\right)^{2}}, \\
\nu_{K} & =N_{k}^{ \pm} e_{ \pm K}^{k}, \\
\lambda_{K L} & =q_{k l}^{ \pm} e_{ \pm K}^{k} e_{ \pm L}^{l},
\end{aligned}
$$


where

$$
N_{ \pm}^{\perp}=N_{k}^{ \pm} m_{ \pm}^{k}, \quad e_{ \pm K}^{k}=\frac{\partial y_{ \pm}^{k}}{\partial \xi^{K}},
$$

and $m_{ \pm}^{k}$ is the unit normal vector to $\Sigma \cap S$ tangent to $S$ and oriented from $S^{-}$to $S^{+}$. From this definition, it follows that

$$
\delta m_{ \pm}^{k}=\frac{1}{2} m_{k}^{ \pm} m_{ \pm}^{r} m_{ \pm}^{s} \delta q_{r s}^{ \pm} .
$$

Using the decomposition (70), we can write for the integrand of the volume terms in Eq. (108):

$$
G_{0}^{0}=-\sqrt{q}\left(N \frac{G^{\perp \perp}}{\sqrt{|g|}}+N^{k} \frac{G_{k}^{\perp}}{\sqrt{|g|}}\right) ;
$$

observe that the R. H. S. is invariant with respect to transformations of coordinates $y^{k}$. The form of $G^{\perp \perp}$ and $G_{k}^{\perp}$ is well-known (cf. [9])

$$
\begin{aligned}
\frac{\sqrt{q}}{\sqrt{|g|}} G^{\perp \perp} & =\frac{2 \pi^{k l} \pi_{k l}-\pi^{2}}{4 \sqrt{q}}-\frac{\sqrt{q}}{2} R^{(3)}, \\
\frac{\sqrt{q}}{\sqrt{|g|}} G_{k}^{\perp} & =-\pi_{k \mid l}^{l},
\end{aligned}
$$

where $R^{(3)}$ is the curvature scalar of the metric $q_{k l}$.

Within $\Sigma$ and $\Sigma^{+}$, an analogous decomposition yields for the surface terms:

$$
T_{s 0}^{0}=-\frac{\sqrt{\lambda}}{\sqrt{|\gamma|}}\left(\nu T_{s}^{\perp \perp}+\nu^{K} T_{s K}^{\perp}\right),
$$

and

$$
\left[Q_{0}^{0}\right]=-\frac{\sqrt{\lambda}}{\sqrt{|\gamma|}}\left(\nu\left[Q^{\perp \perp}\right]+\nu^{K}\left[Q_{K}^{\perp}\right]\right) .
$$

$T_{s 0}^{0}$ can be expressed by means of the canonical variables $z^{A}, p_{A}, \lambda_{K L}, \nu$ and $\nu_{K}$ in a way parallel to Sec. 2.1.4: the formulas are independent of the dimension of spacetime. In general, the form is only implicit. However, the dependence of $T_{s 0}^{0}$ on $\lambda_{K L}, \nu$ and $\nu_{K}$ can be inferred from the relation

$$
T_{s}^{\alpha \beta}=-2 \frac{\partial\left(-T_{s 0}^{0}\right)}{\partial \gamma_{\alpha \beta}}
$$

analogous to Eq. (27). It follows that

$$
\frac{\partial T_{s 0}^{0}}{\partial \nu}=-\frac{\sqrt{\lambda}}{\sqrt{|\gamma|}} T_{s}^{\perp \perp},
$$




$$
\frac{\partial T_{s 0}^{0}}{\partial \nu^{K}}=\frac{\sqrt{\lambda}}{\sqrt{|\gamma|}} T_{s K}^{\perp}
$$

and

$$
\frac{\partial T_{s 0}^{0}}{\partial \lambda_{K L}}=\frac{1}{2} T_{s}^{K L}
$$

In particular, $\frac{\sqrt{\lambda}}{\sqrt{|\gamma|}} T_{s}^{\perp \perp}$ and $\frac{\sqrt{\lambda}}{\sqrt{|\gamma|}} T_{s K}^{\perp}$ are both independent of $\nu$ and $\nu_{K}$.

In an analogous way as in Sec. 2.1.4 we obtain easily

$$
\frac{1}{n_{s}^{2}}\left(\frac{j_{s}^{0}}{\sqrt{\lambda}}\right)^{2}=1+\frac{\lambda^{K L} z_{K}^{A} z_{L}^{B} p_{A} p_{B}}{\left(j_{s}^{0}\right)^{2} \rho_{s}^{\prime 2}}
$$

and

$$
T_{s 0}^{0}=-\nu\left(\frac{\sqrt{\lambda} n_{s}}{\rho_{s}^{\prime}\left(j_{s}^{0}\right)^{2}} \lambda^{K L} z_{K}^{A} z_{L}^{B} p_{A} p_{B}+\sqrt{\lambda} \rho_{s}\right)-\nu^{K} z_{K}^{A} p_{A} .
$$

For a dust shell, we have

$$
\frac{1}{n_{s}^{2}} \frac{j_{s}^{0}}{\sqrt{\lambda}}=1+\frac{\lambda^{K L} z_{K}^{A} z_{L}^{B} p_{A} p_{B}}{\mu^{2}\left(j_{s}^{0}\right)^{2}}
$$

and

$$
T_{s 0}^{0}=-\nu \sqrt{\mu^{2}\left(j_{s}^{0}\right)^{2}+\lambda^{K L} z_{K}^{A} z_{L}^{B} p_{A} p_{B}}-\nu^{K} z_{K}^{A} p_{A} .
$$

Eq. (116) then yields

$$
T_{s K L}=\nu \frac{p_{A} p_{B} z_{K}^{A} z_{L}^{B}}{\sqrt{\mu^{2}\left(j_{s}^{0}\right)^{2}+\lambda^{K L} z_{K}^{A} z_{L}^{B} p_{A} p_{B}}} .
$$

The form of $\left[Q_{0}^{0}\right]$ can be given explicitly. Let us observe (cf. [14]) that the normals $\tilde{m}, n$ and $m$ are related by

$$
\begin{aligned}
\tilde{m}^{\mu} & =n^{\mu} \sinh \alpha+m^{\mu} \cosh \alpha, \\
\tilde{n}^{\mu} & =n^{\mu} \cosh \alpha+m^{\mu} \sinh \alpha ;
\end{aligned}
$$

recall that $n$ is the normal to $S$ in $M, m$ is the normal to $S \cap \Sigma$ in $S$ ( $m$ is orthogonal to $n), \tilde{n}$ is the normal to $S \cap \Sigma$ in $\Sigma$ and $\tilde{m}$ is the normal to $\Sigma$ in $M$ ( $\tilde{n}$ is orthogonal to $\tilde{m})$. A simple calculation then confirms that the corresponding second fundamental forms $L_{\alpha \beta}, K_{k l}$ and $l_{K L}$ of $\Sigma$ in $M, S$ in $M$ and $\Sigma \cap S$ in $S$, respectively, satisfy the relations

$$
\begin{gathered}
L_{\alpha \beta} e_{K}^{\alpha} e_{L}^{\beta}=-K_{k l} e_{K}^{k} e_{L}^{l} \sinh \alpha+l_{K L} \cosh \alpha, \\
L_{\alpha \beta} \tilde{n}^{\alpha} e_{L}^{\beta}-K_{k l} m^{k} e_{L}^{l}=-\alpha_{, L} .
\end{gathered}
$$

It follows that

$$
\frac{1}{\sqrt{|\gamma|}} Q^{\perp \perp}=\frac{1}{\sqrt{q}} \pi^{\perp \perp} \sinh \alpha-l \cosh \alpha
$$




$$
\frac{1}{\sqrt{|\gamma|}} Q_{K}^{\perp}-\frac{1}{\sqrt{q}} \pi_{K}^{\perp}=\alpha_{, K}
$$

Hence,

$$
Q_{0}^{0}=-\nu\left(\sqrt{\lambda} \tilde{\pi}^{\perp \perp} \sinh \alpha-\sqrt{\lambda} l \cosh \alpha\right)-\nu^{K}\left(\sqrt{\lambda} \tilde{\pi}_{K}^{\perp}+\sqrt{\lambda} \alpha_{, K}\right),
$$

where

$$
l=\lambda^{K L} m_{k \mid l} e_{K}^{k} e_{L}^{l}=q^{k l} m_{k \mid l}
$$

depends only on $q_{k l}$ and its first derivatives. We use also the abbreviations

$$
\tilde{\pi}^{\perp \perp}=\frac{\pi^{k l}}{\sqrt{q}} m_{k} m_{l}, \quad \tilde{\pi}_{K}^{\perp}=\frac{\pi^{k l}}{\sqrt{q}} q_{l r} m_{k} e_{K}^{r}, \quad \tilde{\pi}_{K L}=\frac{\pi^{k l}}{\sqrt{q}} e_{K}^{k} e_{L}^{l},
$$

where

$$
e_{K}^{k}:=\frac{\partial y^{k}}{\partial \eta^{K}}
$$

Finally, the complete Hamiltonian reads

$$
\begin{aligned}
\check{\mathcal{H}} & =\frac{1}{16 \pi \mathrm{G}} \int_{S^{-}} d^{3} y\left\{N\left(\frac{2 \pi^{k l} \pi_{k l}-\pi^{2}}{2 \sqrt{q}}-\sqrt{q} R^{(3)}\right)+N^{k}\left(-2 \pi_{k \mid l}^{l}\right)\right\} \\
& +\frac{1}{16 \pi \mathrm{G}} \int_{S^{+}} d^{3} y\left\{N\left(\frac{2 \pi^{k l} \pi_{k l}-\pi^{2}}{2 \sqrt{q}}-\sqrt{q} R^{(3)}\right)+N^{k}\left(-2 \pi_{k \mid l}^{l}\right)\right\} \\
& -\frac{1}{8 \pi \mathrm{G}} \int_{S \cap \Sigma} d^{2} \eta \sqrt{\lambda}\left(\nu\left[\tilde{\pi}^{\perp \perp} \sinh \alpha-l \cosh \alpha\right]+\nu^{K}\left[\tilde{\pi}_{K}^{\perp}+\alpha_{, K}\right]\right) \\
& -\int_{S \cap \Sigma} d^{2} \eta T_{s 0}^{0}+\frac{1}{8 \pi \mathrm{G}} \int_{S \cap \Sigma^{+}} d^{2} \eta L_{0}^{0} .
\end{aligned}
$$

The surface term at $\Sigma^{+}$is left unchanged; it has to be transformed according to the control mode used and/or shifted to infinity.

The surface super-Hamiltonian $\mathcal{H}_{s}$ and the surface supermomentum $\mathcal{H}_{s K}$ at the shell are given by

$$
\begin{aligned}
\mathcal{H}_{s} & =-\frac{1}{8 \pi \mathrm{G}}\left[\tilde{\pi}^{\perp \perp} \sinh \alpha-l \cosh \alpha\right]+\tilde{T}_{s}^{\perp \perp}, \\
\mathcal{H}_{s K} & =-\frac{1}{8 \pi \mathrm{G}}\left[\tilde{\pi}_{K}^{\perp}+\alpha_{, K}\right]+\tilde{T}_{s K}^{\perp}
\end{aligned}
$$

here

$$
\begin{aligned}
\tilde{T}_{s}^{\perp \perp} & =\frac{1}{\sqrt{|\gamma|}} T_{s}^{\alpha \beta} \tilde{n}_{\alpha} \tilde{n}_{\beta} \\
\tilde{T}_{s K}^{\perp} & =\frac{1}{\sqrt{|\gamma|}} T_{s}^{\alpha \beta} \tilde{n}_{\alpha} e_{\beta K} .
\end{aligned}
$$


The geometric meaning of the gravitational part of $\mathcal{H}_{s}$ and $\mathcal{H}_{s K}$ can be inferred from Eq. (54):

$$
\begin{aligned}
& \frac{Q^{\perp \perp}}{\sqrt{|\gamma|}}=-L_{\alpha \beta} e_{K}^{\alpha} e_{L}^{\beta} \gamma^{K L}, \\
& \frac{Q_{K}^{\perp}}{\sqrt{|\gamma|}}=-L_{\alpha \beta} \tilde{n}^{\alpha} e_{K}^{\beta} .
\end{aligned}
$$

In particular, $L_{\alpha \beta} e_{K}^{\alpha} e_{L}^{\beta}$ is the second fundamental form of the two-surface $S \cap \Sigma$ corresponding to the normal $\tilde{m}$ (each two-surface has two independent second fundamental forms in the spacetime); hence, the gravity part of the surface super-Hamiltonian is the jump in the (two-)trace of this form.

\subsection{Equations of motion}

In this subsection, we calculate the variation of the Hamiltonian (118) explicitly. In this way, we can check if our method leads to the well-known equations of motion; moreover, we can study the structure of the canonical equations and constraints at the surface $\Sigma$. In varying the Hamiltonian, we must carefully deal with boundary terms.

The variation of the volume integrands can be given the form:

$$
\delta\left(2 G_{0}^{0}\right)=C_{k} \delta N^{k}+C \delta N+a^{k l} \delta q_{k l}+b_{k l} \delta \pi^{k l}+\sqrt{q} B_{\mid k}^{k},
$$

where

$$
\begin{gathered}
C_{k}=-2 \pi_{k \mid l}^{l}, \\
C=\frac{1}{\sqrt{q}}\left(\pi^{k l} \pi_{k l}-\frac{1}{2} \pi^{2}\right)-\sqrt{q} R^{(3)}, \\
a^{k l}=\frac{N}{\sqrt{q}}\left(2 \pi_{m}^{k} \pi^{l m}-\pi \pi^{k l}-\frac{1}{2} \pi^{m n} \pi_{m n} q^{k l}\right. \\
\left.+\frac{1}{4} \pi^{2} q^{k l}\right)+N \sqrt{q}\left(R^{(3) k l}-\frac{1}{2} R^{(3)} q^{k l}\right)+\sqrt{q}\left(N_{\mid m}^{m} q^{k l}-N_{\mid}^{k l}\right)-\mathcal{L}_{\vec{N}} \pi^{k l}, \\
b_{k l}=\frac{N}{\sqrt{q}}\left(2 \pi_{k l}-\pi q_{k l}\right)-\mathcal{L}_{\vec{N}} q_{k l},
\end{gathered}
$$

and

$$
\begin{aligned}
B^{r} & =-\frac{1}{\sqrt{q}}\left(N^{k} \pi^{l r}+N^{l} \pi^{k r}-N^{r} \pi^{k l}\right) \delta q_{k l}-\frac{2}{\sqrt{q}} N_{l} \delta \pi^{l r} \\
& -N\left(q^{k l} \delta \Gamma_{k l}^{r}-q^{k r} \delta \Gamma_{k l}^{l}\right)+\frac{1}{2} N_{, s}\left(q^{r k} q^{s l}+q^{r l} q^{s k}-2 q^{r s} q^{k l}\right) \delta q_{k l}
\end{aligned}
$$


Here, $\mathcal{L}_{\vec{X}}$ is the Lie derivative with respect to the vector field $\vec{X}$. The comparison with the volume term of Eq. (107) yields the well-known canonical form of Einstein equations (cf. 99):

$$
\begin{gathered}
C_{k}=0, \quad C=0, \\
\dot{\pi}^{k l}=-a^{k l}, \quad \dot{q}_{k l}=b_{k l} .
\end{gathered}
$$

The divergence term contributes to the variation of the surface term at $S \cap \Sigma$ by

$$
\frac{1}{16 \pi \mathrm{G}} \int_{S \cap \Sigma} d^{2} \eta \sqrt{\lambda}\left(-B_{+}^{k} m_{k}^{+}+B_{-}^{k} m_{k}^{-}\right)
$$

The following identity can be easily derived

$$
q^{k l} m_{r}\left(\delta \Gamma_{k l}^{r}-\delta_{k}^{r} \Gamma_{l s}^{s}\right)=2 \delta l+2 l^{K L} \delta \lambda_{K L}-\lambda^{K L}\left(m^{k} e_{L}^{l} \delta q_{k l}\right)_{\| K},
$$

where the symbol "\|" denotes the covariant derivative associated with the metric $\lambda_{K L}$ on $S \cap \Sigma$. Using Eq. (120) and the continuity relations (111) and (112), we can rewrite the surface term in the $2+1$ form

$$
\begin{aligned}
\sqrt{\lambda} B^{k} m_{k} & =-\sqrt{\lambda}\left(2 \tilde{\pi}^{\perp \perp} N^{\perp}+\tilde{\pi}^{\perp K} \nu_{K}\right)\left(m^{k} m^{l} \delta q_{k l}\right)-2 \sqrt{\lambda} \tilde{\pi}^{\perp \perp} \nu^{K}\left(e_{K}^{k} m^{l} \delta q_{k l}\right) \\
& -\sqrt{\lambda}\left(\tilde{\pi}^{\perp L} \nu^{K}+\tilde{\pi}^{\perp K} \nu^{L}-N^{\perp} \tilde{\pi}^{K L}+\tilde{\pi}^{\perp \perp} N^{\perp} \lambda^{K L}+\tilde{\pi}^{\perp M} \nu_{M} \lambda^{K L}\right. \\
& \left.+N_{, k} m^{k} \lambda^{K L}-N l^{K L}\right) \delta \lambda_{K L} \\
& +2 \sqrt{\lambda} N \delta l-2 \sqrt{\lambda}\left(N^{\perp} m_{k} m_{l}+\nu_{K} e_{k}^{K} m_{l}\right) \delta \tilde{\pi}^{k l} .
\end{aligned}
$$

The variation of the surface term $\left[Q_{0}^{0}\right]$ can be written in the following way

$$
\delta Q_{0}^{0}=\delta \nu\left(-\frac{\sqrt{\lambda}}{\sqrt{|\gamma|}} Q^{\perp \perp}\right)-\delta \nu^{K}\left(\frac{\sqrt{\lambda}}{\sqrt{|\gamma|}} Q_{K}^{\perp}\right)+\text { Rest. }
$$

Similarly, using Eqs. (114), (115) and (116), we obtain that

$$
\begin{aligned}
\delta T_{s 0}^{0} & =\delta \nu\left(-\frac{\sqrt{\lambda}}{\sqrt{|\gamma|}} T_{s}^{\perp \perp}\right)-\delta \nu^{K}\left(\frac{\sqrt{\lambda}}{\sqrt{|\gamma|}} T_{s K}^{\perp}\right)+\frac{1}{2} T_{s}^{K L} \delta \lambda_{K L} \\
& +\left(\frac{\partial T_{s 0}^{0}}{\partial z^{A}}-\frac{\partial}{\partial \eta^{M}} \frac{\partial T_{s 0}^{0}}{\partial z_{M}^{A}}\right) \delta z^{A}+\frac{\partial T_{s 0}^{0}}{\partial p_{A}} \delta p_{A} .
\end{aligned}
$$

Comparing the first two terms in Eqs. (121) and (123), we obtain the first three Israel's equations:

$$
\left[Q^{\perp \perp}\right]=8 \pi \mathrm{G} T_{s}^{\perp \perp}, \quad\left[Q_{K}^{\perp}\right]=8 \pi \mathrm{G} T_{s K}^{\perp} .
$$

The last two terms in Eq. (123), if compared with the corresponding surface term in Eq. (107) yield the dynamical equations for matter inside the three-dimensional spacetime of the shell surface $\Sigma$ :

$$
\frac{\partial T_{s 0}^{0}}{\partial z^{A}}-\frac{\partial}{\partial \eta^{M}} \frac{\partial T_{s 0}^{0}}{\partial z_{M}^{A}}=\dot{p}_{A}
$$




$$
\frac{\partial T_{s 0}^{0}}{\partial p_{A}} \delta p_{A}=-\dot{z}^{A}
$$

What remains from Eq. (107) can be written as follows

$$
\left[2 \operatorname{Rest}-\sqrt{\lambda} B^{k} m_{k}\right]=\left(8 \pi \mathrm{G} T_{s}^{K L}-\sqrt{\lambda}[\dot{\alpha}] \lambda^{K L}\right) \delta \lambda_{K L}+\sqrt{\lambda} \lambda^{K L} \dot{\lambda}_{K L} \delta[\alpha] .
$$

A somewhat lenghty calculation starting with Eqs. (121) and (117) gives

$$
\begin{aligned}
& \text { 2Rest }-\sqrt{\lambda} B^{k} m_{k}= \\
& \quad-2 \sqrt{\lambda} \tilde{\pi}^{\perp \perp}\left(\nu \sinh \alpha-N^{\perp}\right) m^{k} m^{l} \delta q_{k l}-\sqrt{\lambda}\left\{\tilde{\pi}^{\perp \perp} \lambda^{K L}\left(\nu \sinh \alpha-N^{\perp}\right)\right. \\
& \left.+\tilde{\pi}^{K L} N^{\perp}+l^{K L} N-\nu l \lambda^{K L} \cosh \alpha+\nu^{M} \lambda^{K L} \alpha_{, M}-N_{, k} m^{k} \lambda^{K L}\right\} \delta \lambda_{K L} \\
& \quad+2 \sqrt{\lambda}(\nu \cosh \alpha-N) \delta l-2 \sqrt{\lambda}\left(\nu \sinh \alpha-N^{\perp}\right) m_{k} m_{l} \delta \tilde{\pi}^{k l} \\
& \quad+2 \sqrt{\lambda}\left(-\nu \tilde{\pi}^{\perp \perp} \cosh \alpha+\nu l \sinh \alpha+\nu_{\| K}^{K}\right) \delta \alpha .
\end{aligned}
$$

Substituting this into Eq. (127), we obtain immediately

$$
\begin{gathered}
\nu \sinh \alpha_{ \pm}=N_{ \pm}^{\perp}, \quad \nu \cosh \alpha_{ \pm}=N_{ \pm}, \\
{\left[-\tilde{\pi}^{\perp \perp} \cosh \alpha+l \sinh \alpha\right]=0 .}
\end{gathered}
$$

The remaining equations, simplified by (128) and (129), read

$$
\sqrt{\lambda}\left[-\tilde{\pi}^{K L} N^{\perp}-l^{K L} N+N l \lambda^{K L}+N_{, k} m^{k} \lambda^{K L}+\nu^{M} \lambda^{K L} \alpha_{, M}+\dot{\alpha} \lambda^{K L}\right]=8 \pi \mathrm{G} T_{s}^{K L},
$$

and

$$
\dot{\lambda}=-2 \lambda\left(N \tilde{\pi}^{\perp \perp}-N^{\perp} l-\nu_{\| K}^{K}\right) .
$$

From the point of view of physical (or geometrical) content, Eq. (128) just reproduces the definition of $\alpha$ and it is compatible with the continuity relation (110). The three Eqs. (130) are equivalent to the remaining three Israel's equations. Finally, Eqs. (129) and (131) follow from the continuity relations (110)-(112) and the equation of motion (119). Indeed, taking limit of the second Eq. (119) from both sides towards the shell and projecting the result by $e_{K}^{k} e_{L}^{l}$, we obtain

$$
\dot{\lambda}_{K L}=\left(2 N\left(\tilde{\pi}_{K L}-\frac{1}{2} \tilde{\pi} \lambda_{K L}\right)+N_{K \| L}+N_{L \| K}+2 l_{K L} N^{\perp}\right)_{ \pm}
$$

the expression in the brackets on the R.H.S. must be continuous, hence

$$
\left[-N\left(\tilde{\pi} \lambda_{K L}-2 \tilde{\pi}_{K L}\right)+2 l_{K L} N^{\perp}\right]=0
$$

The trace of Eq. (133) is Eq. (129) and the trace of Eq. (132) is Eq. (131). 
Eqs. (124), (133) and the tracefree part of Eq. (130) can be written in a more symmetric form

$$
\begin{aligned}
{\left[-K_{K L} \sinh \alpha+l_{K L} \cosh \alpha\right] } & =-8 \pi \mathrm{G}\left(\tilde{T}_{s K L}-\frac{1}{2} \tilde{T} \lambda_{K L}\right), \\
{\left[K_{K L} \cosh \alpha-l_{K L} \sinh \alpha\right] } & =0 \\
{\left[\tilde{\pi}_{K}^{\perp}+\alpha_{, K}\right]=-8 \pi \mathrm{G} \tilde{T}_{s K}^{\perp} } &
\end{aligned}
$$

where $K_{K L}=-\tilde{\pi}_{K L}+(1 / 2) \tilde{\pi} \lambda_{K L}$. They give the jumps of the two independent second fundamental forms of the shell 2-surface in the spacetime, one corresponding to the normal $\tilde{n}$ in the direction of the shell motion (continuous), the other to $\tilde{m}$, which is perpendicular to the direction of motion.

From the point of view of the theory of constraint systems, the Eqs. (124), (129), and the tracefree part of Eq. (130) are constraints. The trace of Eq. (130),

$$
[\dot{\alpha}]=4 \pi \mathrm{G} \nu \tilde{T}_{s}^{K L} \lambda_{K L}-\frac{1}{2}\left[-N^{\perp} \tilde{\pi}^{K L} \lambda_{K L}+N l+2 N_{, k} m^{k}+2 \nu^{K} \alpha_{, K}\right],
$$

Eq. (131) and Eqs. (125), (126) are canonical equations. Finally, Eqs. (110)-(112) and $(\overline{128})$ are defining equations of the Hamiltonian system, analogous to fall-off conditions or control conditions.

It seems that some of the constraints are second class. For example, Eq. (129) follows from the variation with respect to $\bar{\alpha}=(1 / 2)\left(\alpha_{+}+\alpha_{-}\right)$, which is a Lagrange multiplier. Eq. (129) contains this Lagrange multiplier; thus, its Poisson bracket with $\pi_{\bar{\alpha}}$, which is the momentum conjugate to $\bar{\alpha}$, and which is also constrained to vanish, is not zero (if we extend the system by this momentum).

Another important observation is that the L. H. S. of Eq. (129) can be smeared only by a function of two variables, because the domain of definition of the L. H. S. is the shell surface. On the other hand, Eq. (129) contains so-called volume quantities, namely $\pi^{k l}$ and $l^{K L}$; a derivative with respect to these variables and the Poisson brackets of these variables result in three-dimensional $\delta$-functions. Thus, the L. H. S. of Eq, (129) cannot be smeared so that it becomes a differentiable function on the phase space. We call such constraints singular. The best way of tackling this constraint may be to solve it for $\bar{\alpha}$ and insert the solution back into the action (cf. [18]). A similar procedure exists hopefully for the two constraints which result from the tracefree part of Eq. (130):

$$
\begin{aligned}
& 8 \pi \mathrm{G}\left(\tilde{T}_{s}^{K L}-\frac{1}{2} \tilde{T}_{s}^{M N} \lambda_{M N} \lambda^{K L}\right)= \\
& \quad\left[-\left(\tilde{\pi}^{K L}-\frac{1}{2} \tilde{\pi}^{M N} \lambda_{M N} \lambda^{K L}\right) \cosh \alpha-\left(l^{K L}-\frac{1}{2} l^{M N} \lambda_{M N} \lambda^{K L}\right) \sinh \alpha\right]
\end{aligned}
$$

they together with Eq. (129) exhaust the singular constraints of our system. It is interesting to observe that the total Hamiltonian is a differentiable function. Although the surface integrals in the Hamiltonian represent two-dimensional smearing 
of some volume quantities, the presence of volume terms and the continuity relations between the surface $\left(\nu, \nu^{K}\right)$ and volume $\left(N, N^{k}\right)$ smearing functions guarantee an effectively three-dimensional smearing of all volume quantities.

These difficulties and the related problem of the Bergmann-Dirac analysis 17 seem to be non-trivial; we will try to tackle them in a future paper.

\section{Acknowledgements}

The authors are indebted to G. Lavrelashvili for carefully reading the manuscript and for checking some equations. P.H. thanks to the Max-Planck-Institut for Gravitationsphysik, Potsdam, where some calculations have been performed, for the nice hospitality and support, to the Tomalla Foundation, Zurich and to the Swiss Nationalfonds for a partial support. J.K. thanks to the Tomalla Foundation, Zurich and to the Polish National Commitee for Science and Research (KBN) for a partial support.

\section{A Proof of Lemma 3}

Let us calculate the delta-function terms in the expression $L_{g}=h^{\mu \nu} R_{\mu \nu}$, where $h^{\mu \nu}$ is defined by

$$
h^{\mu \nu}:=\frac{1}{16 \pi \mathrm{G}}|g|^{1 / 2} g^{\mu \nu} .
$$

For this aim, we have to isolate the second derivative terms. A simple calculation gives

$$
h^{\mu \nu} R_{\mu \nu}=\partial_{\rho}\left(h^{\mu \nu} A_{\mu \nu}^{\rho}\right)+\ldots
$$

where $A_{\mu \nu}^{\rho}$ is defined by

$$
A_{\mu \nu}^{\lambda}:=\Gamma_{\mu \nu}^{\lambda}-\delta_{(\mu}^{\lambda} \Gamma_{\nu) \kappa}^{\kappa}
$$

and the dots represent regular terms. Thus, the delta-function term in $h^{\mu \nu} R_{\mu \nu}$ is given by

$$
h^{\mu \nu}\left[A_{\mu \nu}^{3}\right] \delta\left(x^{3}-x_{0}^{3}\right) .
$$

Thus, we are to prove the following identity

$$
h^{\mu \nu}\left[A_{\mu \nu}^{3}\right]=-\frac{1}{8 \pi \mathrm{G}} \sqrt{|\gamma|}[L],
$$

where $L_{\alpha \beta}$ is the second fundamental form of $\Sigma$ and $L=\gamma^{\alpha \beta} L_{\alpha \beta}$. Observe that the

R.H.S. of (140) is written in a thre-covariant form. From the definition of (139) of the quantity $A_{\mu \nu}^{\rho}$, it follows that

$$
A_{33}^{3}=-\Gamma_{3 \alpha}^{\alpha}
$$




$$
\begin{aligned}
A_{\alpha 3}^{3} & =\frac{1}{2}\left(\Gamma_{3 \alpha}^{3}-\Gamma_{\beta \alpha}^{\beta}\right), \\
A_{\alpha \beta}^{3} & =\Gamma_{\alpha \beta}^{3} .
\end{aligned}
$$

Let $m_{\mu}$ denote the unit normal vector to $\Sigma$ oriented in the direction of inceasing $x^{3}$, that is outward with respect to $V^{-}$:

$$
m_{\mu}=\frac{1}{\sqrt{g^{33}}} \delta_{\mu}^{3}
$$

Then,

SO

$$
L_{\alpha \beta}=m_{\alpha ; \beta}=-\frac{\Gamma_{\alpha \beta}^{3}}{\sqrt{g^{33}}},
$$

$$
A_{\alpha \beta}^{3}=-\sqrt{g^{33}} L_{\alpha \beta}
$$

$h^{\mu \nu}$ is a tensor density, hence

$$
\nabla_{\kappa} h^{\mu \nu}=h_{, \kappa}^{\mu \nu}+\Gamma_{\kappa \lambda}^{\mu} h^{\lambda \nu}+\Gamma_{\kappa \lambda}^{\nu} h^{\mu \lambda}-\Gamma_{\kappa \lambda}^{\lambda} h^{\mu \nu},
$$

but it is a tensor density formed from the components of the metric tensor, thus $\nabla_{\kappa} h^{\mu \nu}=0$ for all $\kappa, \mu, \nu$. Setting $\mu=3, \nu=\kappa=\alpha$ in this equation and using Eqs. (141), (145) and (146), we obtain that

$$
A_{33}^{3}=\frac{h_{, \alpha}^{3 \alpha}-\sqrt{g^{33}} L_{\alpha \beta} h^{\alpha \beta}}{h^{33}} .
$$

Similarly

$$
A_{\alpha 3}^{3}=-\frac{h_{, \alpha}^{33}-2 \sqrt{g^{33}} L_{\alpha \beta} h^{3 \beta}}{2 h^{33}} .
$$

The metric $g_{\mu \nu}(x)$ is continuous across the shell, so will be $h^{\mu \nu}$ and the tangential derivatives of $h^{\mu \nu}$. It follows that

$$
\begin{aligned}
& {\left[A_{33}^{3}\right]=-\frac{g^{\alpha \beta}}{\sqrt{g^{33}}}\left[L_{\alpha \beta}\right],} \\
& {\left[A_{\alpha 3}^{3}\right]=\frac{g^{3 \beta}}{\sqrt{g^{33}}}\left[L_{\alpha \beta}\right],}
\end{aligned}
$$

where we also have substituted for $h^{\mu \nu}$ from (138). Moreover, using Eq. (146), we have that

$$
\left[A_{\alpha \beta}^{3}\right]=-\frac{g^{33}}{\sqrt{g^{33}}}\left[L_{\alpha \beta}\right] .
$$

The Eqs. (148), (149) and (150) imply

$$
h^{\mu \nu}\left[A_{\mu \nu}^{3}\right]=-\frac{1}{8 \pi \mathrm{G}} \sqrt{|g| g^{33}}\left(g^{\alpha \beta}-\frac{g^{3 \alpha} g^{3 \beta}}{g^{33}}\right)\left[L_{\alpha \beta}\right] .
$$


However, the following well-known relation holds:

$$
\gamma^{\alpha \beta}=g^{\alpha \beta}-\frac{g^{3 \alpha} g^{3 \beta}}{g^{33}},
$$

from which, if Eq. (79) is used, the identity (140) follows immediately.

\section{B Proof of Lemma 4}

If we rewrite $R_{0}^{0}$ in terms of the connection,

$$
R_{0}^{0}=g^{0 \mu}\left(\partial_{\rho} \Gamma_{\mu 0}^{\rho}-\partial_{0} \Gamma_{\mu \rho}^{\rho}+\ldots\right),
$$

where the dots represent terms that do not contain second derivatives of the metric, we obtain immediately that

$$
R_{0}^{0}=g^{0 \mu}\left[\Gamma_{\mu 0}^{3}\right] \delta\left(x^{3}-x_{0}^{3}\right)+\ldots
$$

We easily find:

$$
g^{0 \mu}\left[\Gamma_{0 \mu}^{3}\right]=-g^{33} \gamma^{0 \alpha}\left[g_{0 \alpha, 3}\right] .
$$

The following two equations are easily verified:

$$
\begin{aligned}
g_{\alpha \beta, 3} & =\frac{1}{g^{33}} L_{\alpha \beta}, \\
L_{\alpha \beta} & =\frac{1}{|\gamma|}\left(\frac{1}{2} Q \gamma_{\alpha \beta}-Q_{\alpha \beta}\right) .
\end{aligned}
$$

Then, some computation leads to

$$
g^{0 \mu}\left[\Gamma_{0 \mu}^{3}\right]=\frac{\sqrt{g^{33}}}{\sqrt{|\gamma|}}\left(\left[Q_{0}^{0}\right]-\frac{1}{2}[Q]\right) .
$$

Finally, using Eqs. (79) and (151), we obtain Eq. (109) immediately.

\section{References}

[1] V. de la Cruz, J. E. Chase and W. Israel, Phys. Rev. Letters 24 (1970) 423.

[2] C. J. Farrugia and P. Hájíček, Commun. Math. Phys. 68 (1979) 291.

[3] P. Hájíček, B. S. Kay and K. Kuchař, Phys. Rev. D46 (1992) 5439.

[4] E. Farhi, A. H. Guth and J. Guven, Nucl. Phys. B339 (1990) 417. 
[5] P. Kraus and F. Wilczek, Nucl. Phys. B433, 403 (1995).

[6] E. A. Martinez and J. W. York, Jr., Phys. Rev. D40 (1989) 2125.

[7] V. Berezin, Preprint, INR 0916/96, April 1996.

[8] R. Casadio and G. Venturi, Class. Quantum Grav. 13 (1996) 2715.

[9] C. W. Misner, K. S. Thorne and J. A. Wheeler, Gravitation. Freeman, San Francisco, 1973.

[10] R. Dautcourt, Math. Nachr. 27 (1964) 277.

[11] W. Israel, Nuovo Cim. 44B (1966) 1; 48B (1967) 463.

[12] G. Hayward and J. Louko, Phys. Rev. D42 (1990) 4032.

[13] J. Kijowski and W. M. Tulczyjew, A Symplectic Framework for Field Theories, Lecture Notes in Physics Vol. 107, Springer, Berlin, 1979.

[14] J. Kijowski, Gen. Rel. Grav. 29 (1997) 307; J. Kijowski, Asymptotic degrees of freedom and gravitational energy, in Proceedings of Journees Relativistes 1983, Pitagora Editrice, Bologna 1985, p. 205-219.

[15] J. Kijowski, A. Smólski and A. Górnicka, Phys. Rev. D41 (1990) 1875.

[16] J. D. Brown, Class. Quantum Grav. 10 (1993) 1579.

[17] J. Isenberg and J. Nester, "Canonical Gravity" in General Relativity and Gravitation. One Hundred Years After the Birth of Albert Einstein. Ed. by A .Held. Plenum Press, New York, 1980.

[18] M. Henneaux and C. Teitelboim, Quantization of Gauge Systems. Princeton University Press, Princeton, 1992.

[19] F. J. Belinfante, Physica 6 (1939) 887.

[20] F. J. Belinfante, Physica 7 (1940) 449.

[21] L. Rosenfeld, Acad. Roy. Belg. 18 (1940) 1. 